\title{
A decision-theoretic rough set model with $q$-rung orthopair fuzzy information and its application in stock investment evaluation
}

\author{
Guolin Tang ${ }^{a}$, Francisco Chiclana $^{b, c}$, Peide Liu $^{a^{*}}$ \\ ${ }^{a}$ School of Management Science and Engineering, Shandong University of Finance and Economics, Shandong, China \\ ${ }^{b}$ Institute of Artificial Intelligence, De Montfort University, Leicester, UK \\ ${ }^{c}$ Andalusian Research Institute on Data Science and Computational Intelligence, University of Granada, Granada, Spain \\ *Corresponding author: Tel: +86-531-82222188, E-mail:Peide.liu@gmail.com
}

\begin{abstract}
Stock investment is characterized by high risk and massive profit, so it is necessary to propose a scientific and accurate stock assessment and selection method for avoiding investment risks and obtaining high returns. Stock investment evaluation and selection can be regarded as a three-way decision (3WD) problem. Decision-theoretic rough sets (DTRSs) are an excellent tool to cope with 3 WDs under risks and uncertainty. Due to the increasing complexity and high uncertainty of decision environments, the loss functions involved in DTRSs are not always expressed with real numbers. As a novel generalized form of Pythagorean fuzzy sets (PFSs) and intuitionistic fuzzy sets (IFSs), $q$-rung orthopair fuzzy sets ( $q$-ROFSs) depict uncertain information more widely and flexibly. Thus, it is a significant innovation to combine $q$-ROFSs with DTRSs and construct a new 3WD model for stock investment evaluation. More specifically, we first extend $q$-rung orthopair fuzzy numbers ( $q$-ROFNs) to DTRSs, which can offer a novel illustration for loss functions. Then, we establish a novel $q$-rung orthopair fuzzy DTRS ( $q$-ROFDTRS) model and explore some fundamental properties of the expected losses. Additionally, we propose two methods to handle $q$-ROFNs and obtain 3 WDs. These two methods are compared, and their characteristics and applicability are analysed. Finally, a practical case concerning stock investment evaluation is supplied to illustrate the effectiveness and the superiority of the developed approaches over existing methods.
\end{abstract}

Keywords: Decision making, Decision-theoretic rough sets, $q$-Rung orthopair fuzzy sets, Loss function

\section{Introduction}

Stock investment refers to the investment behaviour and investment process by which investors purchase stocks to obtain dividends and capital gains. In recent years, with the rapid development of China's securities market, stock investment has become an important channel for enterprises' direct investment and personal investment and financing. Because there are many factors involved in the analysis of stock investment decision making and the stock market is a very complicated system, it is necessary and meaningful to choose a scientific and reasonable investment decision-making method for avoiding investment risks and obtaining high returns. For stock investment evaluation and selection, if investors accept a bad stock, it could result in investment losses; in contrast, if investors reject a good stock, they might miss investment chances. In fact, the non-commitment decision is also a novel added option. In this situation, it is hard for investors to make a choice, while they do not want to lose some opportunities. The non-commitment decision requires investors to collect much more information to make the choice. Thus, the stock investment evaluation problem can be regarded as a three-way decision (3WD) problem. In practice, for the stock investment evaluation problem, most of the 
assessment information is unknown and many factors are influenced by high uncertainty. Consequently, traditional fuzzy sets and their extensions, such as intuitionistic fuzzy sets (IFSs) [5] and Pythagorean fuzzy sets (PFSs) [52], might be inappropriate to deal with practical cases. In this situation, $q$-rung orthopair fuzzy sets ( $q$-ROFSs) [51] could be regarded as a feasible tool to cope with high uncertainty because they can maximize the accuracy and integrity of fuzzy information.

The notion of $q$-ROFSs was presented by Yager [51], whose membership degree (MD), $t(Q R)$ and non-membership degree (NMD), $f(Q R)$ satisfy the restriction $(t(Q R))^{q}+(f(Q R))^{q} \leq 1$. Obviously, $q$-ROFSs are a generalized form of IFSs $(q=1)$ [5, 6, 45-47] and PFSs [52] $(q=2)$. Moreover, $q$-ROFSs can be used to describe larger and more complex fuzzy information because the value of the flexible parameter $q$ can be dynamically adjusted to decide the information expression range. Therefore, compared with IFSs and PFSs, $q$-ROFSs are more general and flexible. To better understand $q$-ROFSs, we provide the following case. Suppose that a decision maker provides an assessment value $(0.7,0.9)$. Then, because $0.7+0.9=1.6>1$ and $0.7^{2}+0.9^{2}=1.3>1$, the assessment value is not possible to be used in IFSs and PFSs contexts. However, it is available for $q$-ROFSs, where $q \geq 4$ because $0.7^{4}+0.9^{4}$ $=0.8962<1$. It is apparent that the $q$-ROFSs include extra uncertainties, while IFSs and PFSs do not, and are typically able to adapt to higher levels of uncertainty. Therefore, $q$-ROFSs are easy to use in practical management application fields.

DTRSs were presented by Yao et al. [59] by combining the rough sets theory [38] with the Bayesian decision procedure [11] to offer, a logical semantic illustration and the corresponding risk factors given a decision-making problem [23]. They can generate three kinds of decision rules through the minimum of the expected risk, namely, positive decisions, boundary decisions and negative decisions [57]. DTRSs are now one of the most famous representative decision-making approaches present in modern decision theory due to their powerful ability to cope with 3WD problems under risks and uncertainty. Since their introduction, DTRSs have developed very rapidly and have been successfully implemented in different domains, such as discriminant analysis [33], risk decision analysis [25], government decision analysis [34], information filtering [22], medical care product investment [16], cluster analysis [60], feature selection [41] and image segmentation [12]. Additionally, to deal with more complex 3WD problems, some modified versions of DTRSs have been proposed. For instance, $\mathrm{Li}$ and $\mathrm{Xu}$ [21] constructed two types of double-quantitative DTRSs models for handling issues that need to capture absolute and relative quantitative information. Then, Yu et al. [61] presented a multi-granulation rough method based on DTRSs and graded rough sets. Jia and Liu [13] developed a novel model by combining 3 WDs and multiple attribute decision making. To effectively integrate the wisdom of several experts, Liang et al. [26] presented group decision-making-based 3WDs by utilizing the principle of justifiable granularity. Additionally, in a realistic decision procedure, a vital issue is how to represent reasonably and accurately the loss function involved in the DTRSs. In practical decision-making situations, people may find it difficult to denote the loss function using crisp values due to the complexity and insufficiency of the available data. For this reason, Liang and Liu [23-25], Liang et al. [30, 31], Zhong and Zhang [62], Zhang [63], Abdel-Basset et al. [1], and Liang et al. [29] extended hesitant fuzzy (HF) sets, interval-valued fuzzy (IVF) sets, intuitionistic fuzzy (IF) sets, Pythagorean fuzzy (PF) sets, dual HF (DHF) sets, generalized trapezoidal fuzzy (GTF) sets, IF soft 
(IFS) sets, neutrosophic fuzzy (NF) sets and interval type-2 fuzzy (IT2F) sets to DTRSs, respectively, and established the corresponding DTRS models.

Although many studies have extended the DTRSs to a variety of fuzzy settings, little attention has been paid to the extension of the DTRSs into $q$-ROFSs settings. As described above, for the stock investment evaluation problem, most of the assessment information is unknown and many factors are influenced by high uncertainty. As a result, traditional fuzzy sets and their extensions, such as IFSs and PFSs, may be insufficient to deal with real-world cases due to the increasing uncertainty of the stock investment evaluation problem, and the $q$-ROFSs can be utilized instead. Simultaneously, DTRSs are an excellent tool to cope with decision-making problems under risks and uncertainty. Therefore, it is justifiable to study the extended DTRS model for stock investment selection within the context of $q$-ROFSs. Motivated by this idea, we extend the DTRS model to accommodate the $q$-ROFS environment and further propose novel 3WDs-DTRSs for solving real-life stock investment selection problems. Specifically, we first establish a $q$-rung orthopair fuzzy DTRS ( $q$-ROFDTRS) model using the loss functions involved in DTRSs and $q$-ROFSs. Then, some desirable features of the expected losses are explored. In addition, from different angles, we propose two methods for obtaining 3WDs with the novel $q$-ROFDTRS model. At the same time, we investigate the performances of these two techniques and summarize their corresponding features. Finally, to illustrate the proposed 3WD methods, a practical case concerning stock investment evaluation is analysed.

To do so, the organization of this article is offered as follows: Section 2 recalls some related literature about investment evaluation and selection. Section 3 concentrates on basic knowledge of $q$-ROFSs. Section 4 establishes novel DTRS models and discusses their properties within the context of $q$-ROFSs. Section 5 proposes two approaches for obtaining 3WDs with the established $q$-ROFDTRS model. Section 6 gives a practical case to delineate the usefulness of the developed methods. Section 7 concludes the study.

\section{Literature review}

Recently, a subject of investment evaluation and selection has been heavily studied in the related literature. Briefly, these include stocks, credit, energy resources, wind power projects, real estate and expressways. These studies can be roughly classified into three categories: the studies based on multiple attribute decision-making methods, the studies based on machine learning techniques and the studies based on three-way decision (3WD) models.

The first category is based on multiple attribute decision-making (MADM) methods, including the data envelopment analysis (DEA), technique for order preference by similarity to ideal solution (TOPSIS), preference ranking organization method for enrichment evaluation (PROMETHEE), analytical network process (ANP), visekriterijumska optimizacija i kompromisno resenje (VIKOR) and an acronym in Portuguese for Interactive Multi-criteria Decision Making (TODIM). For example, Chen [7] used the DEA technique to explore stock selection. Ho and Oh [15] presented an assessment approach to deal with the stock investment problem in Taiwan based on DEA and the analytic hierarchy process (AHP). These two methods could derive efficiency scores of stock companies, but they do not fit properly with the application [4]. To address this issue, Amin and Hajjami [4] used a combined 
ordered weighted averaging and DEA method for stock selection. Additionally, Lim et al. [32] developed a DEA cross-efficiency evaluation for stock portfolio selection in the Korean stock market. Albadvi [2] used the PROMETHEE method to select the superior stocks for investment. Lee et al. [17] applied the ANP model to select stock based on the Gordon technique. Shen et al. [40] presented a novel decision-making method for glamour stock selection based on the ANP method, DEMATEL technique and VIKOR method. However, most of the above decision-making methods use crisp values to describe assessment information. Thus, they cannot express the insufficient physical knowledge. To handle the imprecision of human cognition, Hatami-Marbini and Kangi [14] proposed an extended fuzzy TOPSIS decision method for the Tehran stock exchange. Li et al. [20] discussed the ranking of stocks by integrating TODIM and dual hesitant fuzzy linguistic elements (DHFLEs), whose prominent feature is that the decision maker's psychological behaviour is considered. Although these MADM methods rank stocks from the highest to the lowest score, they could not inform the decision maker regarding how to choose the action for each stock.

The second category is based on machine learning techniques, among which the most popular are back propagation (BP) neural networks, the convolutional neural network (CNN), deep learning and the multi-filters neural network (MFNN) model. For example, Wu et al. [48] applied BP neural networks model to evaluate the Chinese stock market. Cao and Wang [9] established an investment stock selection model based on principal component analysis (PCA), the BP neural network and a stock selection analysis method. Wu et al.'s method [48] and Cao and Wang's method [9] have good self-learning ability and strong self-adaptation ability, but they have two limitations, namely, inability to escape the local optimum and slowness in convergence [18]. To overcome these drawbacks, Qiu et al. [39] presented a hybrid model for stock investment based on the BP, genetic algorithm and simulated annealing approaches. The results displayed that the hybrid model enhances prediction accuracy notably and outperforms the classical BP neural networks. To further enhance the accuracy of networks, Chen et al. [8] designed a financial quantitative investment model using deep learning and CNN approaches. Xie et al. [49] built an automated investment model to forecast stocks' price based on deep learning. Note that both Chen et al.'s method [8] and Xie et al.'s method [49] could predict the ups and downs of stock index futures. Long et al. [37] developed an MFNN model for stock price prediction based on deep learning. The results displayed that the MFNN model outperforms single-structure (long short-term memory, recurrent, and convolutional) networks with regard to the stability, accuracy, and profitability. These approaches have good performances and are able to deal with large amounts of data. Nevertheless, most of these methods are based on minimizing the empirical risk, which has the problem of ensuring that the empirical risk minimization shall also minimize the expected risk when a small training set is employed [42].

The last category is based on 3WD models. DTRSs are leading models in the application of 3WDs on investment evaluation owing to their remarkable capability of handling decision-making problems under risks and uncertainty. For example, Liang et al. [27] presented a triangular fuzzy DTRS model for investment evaluation based on DTRSs, triangular fuzzy numbers and particle swarm optimization. Liang and Liu [24] presented a dynamic 3WD model for the investment decisions based on dynamic intuitionistic fuzzy aggregation operators and DTRSs. Liang et al. [31] presented a novel 3WD model 
for investment evaluation based on DTRSs, Pythagorean fuzzy sets and the TOPSIS method. These models can inform the decision maker regarding how to choose the action for each alternative and provide the corresponding semantic explanations. Additionally, they do not rely on the prior knowledge of the decision data, but automatically make the decisions based on the intrinsic characters of the datasets. An overview of the existing literature on investment evaluation is given in Table 1.

Table 1. Summary of some related literature on investment evaluation.

\begin{tabular}{|c|c|c|c|}
\hline Category & Method & Literature & Characteristics \\
\hline \multirow[t]{6}{*}{$\begin{array}{l}\text { MADM } \\
\text { methods }\end{array}$} & DEA & $\begin{array}{l}\text { Amin and Hajjami [4], Chen [7], } \\
\text { Ho and Oh [15], Lim et al. [32] }\end{array}$ & \multirow{6}{*}{$\begin{array}{l}\text { These methods rank stocks from the highest to the } \\
\text { lowest score, but they could not inform the decision } \\
\text { maker on how to choose the action for each stock. }\end{array}$} \\
\hline & PROMETHEE & Albadvi [2] & \\
\hline & ANP & Lee et al. [17] & \\
\hline & VIKOR & Shen et al. [40] & \\
\hline & Fuzzy TOPSIS & Hatami-Marbini and Kangi [14] & \\
\hline & Fuzzy TODIM & Li et al. [20] & \\
\hline \multirow{5}{*}{$\begin{array}{l}\text { Machine } \\
\text { learning } \\
\text { techniques }\end{array}$} & $\mathrm{BP}$ & Wu et al. [48], Cao and Wang [9] & \multirow{5}{*}{$\begin{array}{l}\text { These approaches are able to deal with large amounts } \\
\text { of data. However, it is hard for these approaches to } \\
\text { ensure that the empirical risk minimization shall also } \\
\text { minimize the expected risk when having a small } \\
\text { training set. }\end{array}$} \\
\hline & $\mathrm{CNN}$ & Chen et al. [8] & \\
\hline & Deep learning & Xie et al. [49] & \\
\hline & MFNN model & Long et al. [37] & \\
\hline & Hybrid model & Qiu et al. [39] & \\
\hline 3WD models & Fuzzy DTRSs & $\begin{array}{l}\text { Liang et al. [27], } \\
\text { Liang and Liu [24], } \\
\text { Liang et al. [31] }\end{array}$ & $\begin{array}{l}\text { These models inform the decision maker on how to } \\
\text { choose the action for each alternative. Additionally, } \\
\text { they do not rely on the prior knowledge of the decision } \\
\text { data and can generate three corresponding actions } \\
\text { through the minimum of the expected risk. }\end{array}$ \\
\hline
\end{tabular}

From Table 1, we observe that fuzzy sets have been used to solve investment evaluation problems. Additionally, compared with the existing MADM methods and machine learning techniques used in investment evaluation, the main advantages of the DTRSs are that the models inform the decision maker on how to choose the action for each alternative and can generate three corresponding actions through the minimum of the expected risk without the prior knowledge of the decision data. However, no attention has been paid to the extension of DTRSs into the $q$-ROF setting to deal with stock investment evaluation and selection problems. Thus, it is necessary and meaningful to combine DTRSs with their evaluation value within the context of the $q$-ROF setting, which can not only improve the model ability to attain high uncertainty but also handle the stock investment problem with imprecise and uncertain decision information. Therefore, the contribution of this paper is that we extend the DTRS model to accommodate the $q$-ROFSs environment and further propose novel 3WDs-DTRSs for solving the real-life stock investment evaluation problem.

\section{3. $q$-Rung orthopair fuzzy sets ( $q$-ROFSs)}

In the following, we shall introduce some basic concepts of $q$-ROFSs [51], which generalize the concepts of IFSs [5, 6] and PFSs [52].

Definition 1. [51]. A $q$-ROFS $Q R$ in a fixed set $Z$ is expressed by 


$$
Q R=\left\{\left\langle z, t_{Q R}(z), f_{Q R}(z)\right\rangle \mid z \in Z\right\},
$$

where $t_{Q R}(z)$ and $f_{Q R}(z)$ are the MD and the NMD of element $z \in Z$ to the $q$-ROFS $Q R$, respectively, with the conditions that $t_{Q R}: Z \rightarrow[0,1], f_{Q R}: Z \rightarrow[0,1], \quad 0 \leq\left(t_{Q R}(z)\right)^{q}+\left(f_{Q R}(z)\right)^{q} \leq 1$. The degree of indeterminacy of element $z \in Z$ belonging to the $q$-ROFS $Q R$ is $\varsigma_{Q R}(z)=\left(1-\left(t_{Q R}(z)\right)^{q}+\left(f_{Q R}(z)\right)^{q}\right)^{1 / q}$, where $q \geq 1$. For simplicity, $Q R=\langle t(Q R), f(Q R)\rangle$ is called a $q$-rung orthopair fuzzy number ( $q$-ROFN).

The main differences of intuitionistic fuzzy number (IFN), Pythagorean fuzzy number (PFN) and $q$-ROFN space ranges are displayed in Figure 1.

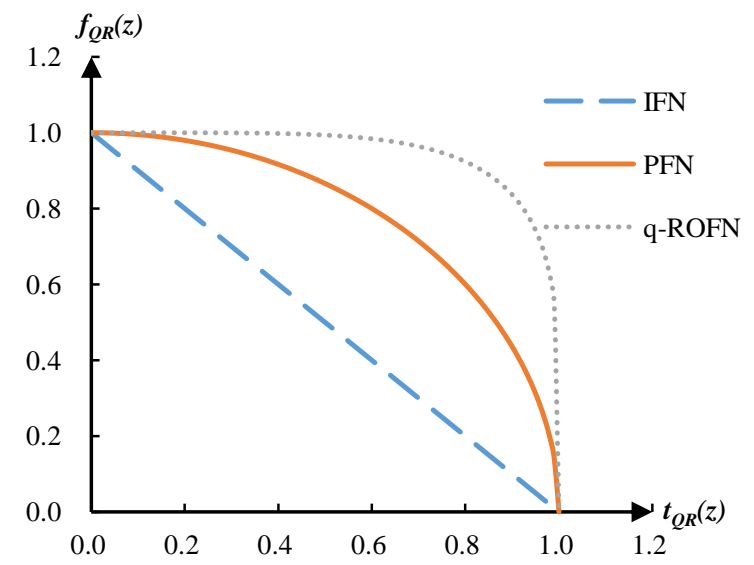

Figure 1. Comparison of the space ranges of IFN, PFN and $q$-ROFN.

Definition 2 [51]. Suppose that $Q R_{1}=\left\langle t\left(Q R_{1}\right), f\left(Q R_{1}\right)\right\rangle$ and $Q R_{2}=\left\langle t\left(Q R_{2}\right), f\left(Q R_{2}\right)\right\rangle$ are two $q$-ROFNs; their operations are defined as follows:

(1) $Q R_{1} \oplus Q R_{2}=\left\langle\left(t\left(Q R_{1}\right)^{q}+t\left(Q R_{2}\right)^{q}-t\left(Q R_{1}\right)^{q} t\left(Q R_{2}\right)^{q}\right)^{1 / q}, f\left(Q R_{1}\right) f\left(Q R_{2}\right)\right\rangle$,

(2) $Q R_{1} \otimes Q R_{2}=\left\langle t\left(Q R_{1}\right) t\left(Q R_{2}\right),\left(f\left(Q R_{1}\right)^{q}+f\left(Q R_{2}\right)^{q}-f\left(Q R_{1}\right)^{q} f\left(Q R_{2}\right)^{q}\right)^{1 / q}\right\rangle$,

(3) $\lambda Q R_{1}=\left\langle\left(1-\left(1-t\left(Q R_{1}\right)^{q}\right)^{\lambda}\right)^{1 / q}, f\left(Q R_{1}\right)^{\lambda}\right\rangle, \lambda>0$,

where $q \geq 1$.

To rank $q$-ROFNs, the score and the accuracy functions of $q$-ROFNs were defined by Liu and Wang [36] as follows:

Definition 3. Suppose that $Q R_{1}=\left\langle t\left(Q R_{1}\right), f\left(Q R_{1}\right)\right\rangle$ is a $q$-ROFN; then, the score function $S\left(Q R_{1}\right)$ of $Q R_{1}$ is

$$
S\left(Q R_{1}\right)=t\left(Q R_{1}\right)^{q}-f\left(Q R_{1}\right)^{q},
$$

where $q \geq 1$ and $S\left(Q R_{1}\right) \in[-1,1]$.

Definition 4. Suppose that $Q R_{1}=\left\langle t\left(Q R_{1}\right), f\left(Q R_{1}\right)\right\rangle$ is a $q$-ROFN; then, the accuracy function $H\left(Q R_{1}\right)$ of $Q R_{1}$ is 


$$
H\left(Q R_{1}\right)=t\left(Q R_{1}\right)^{q}+f\left(Q R_{1}\right)^{q},
$$

where $q \geq 1$ and $H\left(Q R_{1}\right) \in[0,1]$.

Liu and Wang [36] gave the following ranking technique to compare two $q$-ROFNs:

Definition 5. Suppose that $Q R_{1}=\left\langle t\left(Q R_{1}\right), f\left(Q R_{1}\right)\right\rangle$ and $Q R_{2}=\left\langle t\left(Q R_{2}\right), f\left(Q R_{2}\right)\right\rangle$ are any two $q$-ROFNs.

(1) If $S\left(Q R_{1}\right)>S\left(Q R_{2}\right)$, then $Q R_{1}>Q R_{2}$;

(2) If $S\left(Q R_{1}\right)=S\left(Q R_{2}\right)$ and $H\left(Q R_{1}\right)>H\left(Q R_{2}\right)$, then $Q R_{1}>Q R_{2}$;

(3) If $S\left(Q R_{1}\right)=S\left(Q R_{2}\right)$ and $H\left(Q R_{1}\right)=H\left(Q R_{2}\right)$, then $Q R_{1}=Q R_{2}$.

\section{4. $q$-ROFDTRS model}

We propose a novel model of $q$-ROFDTRSs in accordance with the initial DTRSs and $q$-ROFSs. The novel $q$-ROFDTRS model constitutes two states and three actions. The collection of states is presented by $\Theta=\{D, \bar{D}\}$ expressing that an object $z$ belongs to $D$ and does not belong to $D$, respectively. In the meantime, the collection of actions is presented by $\Delta=\left\{\gamma_{\rho}, \gamma_{\beta}, \gamma_{\eta}\right\}$, where $\gamma_{\rho}, \gamma_{\beta}$ and $\gamma_{\eta}$ indicate three actions when classifying object $z$. These three actions actually correspond to the three regions of rough sets [38], namely, determining $z \in \operatorname{Pos}(D)$, determining $z \in \operatorname{Bou}(D)$ and determining $z \in N e g(D)$. Thus, the $q$-rung orthopair fuzzy ( $q$-ROF) loss function matrix with regard to the risk or cost of actions in the different states is presented in Table 2.

Table 2. $q$-ROF loss function matrix

\begin{tabular}{|c|c|c|}
\hline & $D$ & $\bar{D}$ \\
\hline$\gamma_{\rho}$ & $Q R_{\tau_{\rho p}}=\left(t\left(Q R_{\tau_{\rho p}}\right), f\left(Q R_{\tau_{\rho p}}\right)\right)$ & $Q R_{\tau_{\rho \eta}}=\left(t\left(Q R_{\tau_{\rho \eta}}\right), f\left(Q R_{\tau_{\rho \eta}}\right)\right)$ \\
\hline$\gamma_{\beta}$ & $Q R_{\tau_{\beta p}}=\left(t\left(Q R_{\tau_{\beta p}}\right), f\left(Q R_{\tau_{\beta p}}\right)\right)$ & $Q R_{\tau_{\beta_{\eta}}}=\left(t\left(Q R_{\tau_{\beta_{\eta}}}\right), f\left(Q R_{\tau_{\beta_{\eta}}}\right)\right)$ \\
\hline$\gamma_{\eta}$ & $Q R_{\tau_{\tau_{p}}}=\left(t\left(Q R_{\tau_{\tau_{p}}}\right), f\left(Q R_{\tau_{\tau_{p}}}\right)\right)$ & $Q R_{\tau_{m}}=\left(t\left(Q R_{\tau_{m}}\right), f\left(Q R_{\tau_{m}}\right)\right)$ \\
\hline
\end{tabular}

The loss functions $Q R_{\tau_{\bullet}}(\bullet=\rho, \beta, \eta ; \circ=\rho, \eta)$ of Table 2 represent $q$-ROFNs, and $Q R_{\tau_{\rho \rho}}, Q R_{\tau_{\beta \rho}}$ and $Q R_{\tau_{\eta \rho}}$ indicate the loss degrees with $q$-ROFNs incurred for adopting actions of $\gamma_{\rho}, \gamma_{\beta}$ and $\gamma_{\eta}$, respectively, when an object belongs to $D$. Analogously, $Q R_{\tau_{\rho \eta}}, Q R_{\tau_{\beta \eta}}$ and $Q R_{\tau_{\eta \eta}}$ are the loss degrees with $q$-ROFNs incurred for adopting the same actions when the object belongs to $\bar{D}$. Based on the characteristics of $q$-ROFNs and the semantics of DTRSs, we can obtain

$$
\begin{aligned}
& t\left(Q R_{\tau_{\rho \rho}}\right) \leq t\left(Q R_{\tau_{\beta \rho}}\right)<t\left(Q R_{\tau_{\eta \rho}}\right), \\
& f\left(Q R_{\tau_{\eta \rho}}\right)<f\left(Q R_{\tau_{\beta \rho}}\right) \leq f\left(Q R_{\tau_{\rho \rho}}\right), \\
& t\left(Q R_{\tau_{\eta \eta}}\right) \leq t\left(Q R_{\tau_{\beta \eta}}\right)<t\left(Q R_{\tau_{\rho \eta}}\right), \\
& f\left(Q R_{\tau_{\rho \eta}}\right)<f\left(Q R_{\tau_{\beta \eta}}\right) \leq f\left(Q R_{\tau_{\eta \eta}}\right) .
\end{aligned}
$$

Additionally, the following constraints also need to be added:

$$
t\left(Q R_{\tau_{\rho \rho}}\right)^{q}+f\left(Q R_{\tau_{\rho \rho}}\right)^{q} \leq 1
$$




$$
\begin{aligned}
& t\left(Q R_{\tau_{\beta \rho}}\right)^{q}+f\left(Q R_{\tau_{\beta \rho}}\right)^{q} \leq 1, \\
& t\left(Q R_{\tau_{\eta \rho}}\right)^{q}+f\left(Q R_{\tau_{\eta \rho}}\right)^{q} \leq 1, \\
& t\left(Q R_{\tau_{\rho \eta}}\right)^{q}+f\left(Q R_{\tau_{\rho \eta}}\right)^{q} \leq 1, \\
& t\left(Q R_{\tau_{\beta \eta}}\right)^{q}+f\left(Q R_{\tau_{\beta \eta}}\right)^{q} \leq 1, \\
& t\left(Q R_{\tau_{\eta \eta}}\right)^{q}+f\left(Q R_{\tau_{\eta \eta}}\right)^{q} \leq 1 .
\end{aligned}
$$

If the $q$-ROF loss functions of Table 2 meet formulas (3)-(6), then based on Definitions 3-5, we obtain the following:

$$
\begin{aligned}
& Q R_{\tau_{\rho \rho}} \leq Q R_{\lambda_{\beta \rho}}<Q R_{\lambda_{\eta \rho}}, \\
& Q R_{\tau_{\eta \eta}} \leq Q R_{\tau_{\beta \eta}}<Q R_{\tau_{\rho \eta}} .
\end{aligned}
$$

Expression (13) means that the loss degree of predicting the object $z$ belonging to $D$ in $\operatorname{Neg}(D)$ is larger than that of predicting $z$ in $\operatorname{Bou}(D)$, and the loss degree of predicting $z$ in $\operatorname{Bou}(D)$ is not lower than that of predicting $z$ in $\operatorname{Pos}(D)$. A similar interpretation is associated with (14).

For the Bayesian decision procedure [11], the conditional probability is a basic component. Suppose that $\operatorname{Pob}(D \mid[z])$ and $\operatorname{Pob}(\bar{D} \mid[z])$ are the conditional probabilities of $z$ belonging to $D$ and $\bar{D}$, respectively, where $z$ is depicted by its equivalence class $[z]$. Suppose that $\operatorname{Pob}(D \mid[z])$ and $\operatorname{Pob}(\bar{D} \mid[z])$ are crisp numbers. Therefore, we obtain $\operatorname{Pob}(D \mid[z])+\operatorname{Pob}(\bar{D} \mid[z])=1$.

For $z$, the expected losses $\operatorname{EL}\left(\gamma_{\bullet} \mid[z]\right)(\bullet=\rho, \beta, \eta)$ of taking the relevant actions can be illustrated as follows:

$$
\begin{aligned}
& E L\left(\gamma_{\rho} \mid[z]\right)=Q R_{\tau_{\rho \rho}} \operatorname{Pob}(D \mid[z]) \oplus Q R_{\tau_{\rho \eta}} \operatorname{Pob}(\bar{D} \mid[z]), \\
& E L\left(\gamma_{\beta} \mid[z]\right)=Q R_{\tau_{\beta \rho}} \operatorname{Pob}(D \mid[z]) \oplus Q R_{\tau_{\beta \eta}} \operatorname{Pob}(\bar{D} \mid[z]), \\
& E L\left(\gamma_{\eta} \mid[z]\right)=Q R_{\tau_{\eta \rho}} \operatorname{Pob}(D \mid[z]) \oplus Q R_{\tau_{\eta \eta}} \operatorname{Pob}(\bar{D} \mid[z]) .
\end{aligned}
$$

For the expected losses $E L\left(\gamma_{\bullet} \mid[z]\right)(\bullet=\rho, \beta, \eta)$ of (15)-(17), their results are further computed as follows:

$$
\begin{aligned}
& E L\left(\gamma_{\rho} \mid[z]\right) \\
= & \left\langle\left(1-\left(1-t\left(Q R_{\tau_{\rho \rho}}\right)^{q}\right)^{\operatorname{Pob}(D \mid[z])}\left(1-t\left(Q R_{\tau_{\rho \eta}}\right)^{q}\right)^{\operatorname{Pob}(\bar{D} \mid[z])}\right)^{1 / q}, f\left(Q R_{\tau_{\rho \rho}}\right)^{\operatorname{Pob}(D \mid[z])} f\left(Q R_{\tau_{\rho \eta}}\right)^{\operatorname{Pob}(\bar{D} \mid[z])}\right\rangle, \\
& E L\left(\gamma_{\beta} \mid[z]\right) \\
= & \left\langle\left(1-\left(1-t\left(Q R_{\tau_{\beta \rho}}\right)^{q}\right)^{\operatorname{Pob}(D \mid[z])}\left(1-t\left(Q R_{\tau_{\beta \eta}}\right)^{q}\right)^{\operatorname{Pob}(\bar{D} \mid[z])}\right)^{1 / q}, f\left(Q R_{\tau_{\beta \rho}}\right)^{\operatorname{Pob}(D \mid[z])} f\left(Q R_{\tau_{\beta \eta}}\right)^{\operatorname{Pob}(\bar{D} \mid[z])\rangle}\right\rangle \\
& E L\left(\gamma_{\eta} \mid[z]\right) \\
= & \left\langle\left(1-\left(1-t\left(Q R_{\tau_{\eta \rho}}\right)^{q}\right)^{\operatorname{Pob}(D \mid[z])}\left(1-t\left(Q R_{\tau_{\eta \eta}}\right)^{q}\right)^{\operatorname{Pob}(\bar{D} \mid[z])}\right)^{1 / q}, f\left(Q R_{\tau_{\eta \rho}}\right)^{\operatorname{Pob}(D \mid[z])} f\left(Q R_{\tau_{\eta \eta}}\right)^{\operatorname{Pob}(\bar{D} \mid[z])\rangle}\right\rangle
\end{aligned}
$$


Using (18)-(20), the expected losses $E L\left(\gamma_{\bullet} \mid[z]\right)(\bullet=\rho, \beta, \eta)$ can be expressed as shown below:

$$
\begin{aligned}
& E L(\gamma \cdot \mid[z]) \\
= & \left\langle\left(1-\left(1-t\left(Q R_{\tau_{\bullet}}\right)^{q}\right)^{P o b(D \mid[z])}\left(1-t\left(Q R_{\tau_{\bullet_{\eta}}}\right)^{q}\right)^{P o b(\bar{D} \mid[z])}\right)^{1 / q}, f\left(Q R_{\tau_{\bullet \rho}}\right)^{P o b(D[[z])} f\left(Q R_{\tau_{\tau_{\eta}}}\right)^{P o b(\bar{D}[z])}\right\rangle .
\end{aligned}
$$

In what follows, we explore the effect of $t\left(Q R_{\tau_{\omega_{\rho}}}\right)$ and $t\left(Q R_{\tau_{\tau_{\eta}}}\right)$ on the expected losses.

Proposition 1. Suppose that $t(Q R)_{E L(\gamma,[z])}=\left(1-\left(1-t\left(Q R_{\tau_{\bullet}}\right)^{q}\right)^{P o b(D \|[z])}\left(1-t\left(Q R_{\tau_{\bullet}}\right)^{q}\right)^{P o b(\bar{D}[z]])}\right)^{1 / q}$, where $\operatorname{Pob}(D \mid[z])$ and $q$ are constant. Then, $t(Q R)_{E L\left(\gamma_{0}[z]\right)}$ is non-monotonic decreasing with respect to $t\left(Q R_{\tau_{\bullet \rho}}\right)$ and $t\left(Q R_{\tau_{\bullet \eta}}\right)(\bullet=\rho, \beta, \eta)$.

The proof of Proposition 1 is provided in Appendix 1.

Now, we explore the effect of $f\left(Q R_{\tau_{\bullet \rho}}\right)$ and $f\left(Q R_{\tau_{v_{\eta}}}\right)$ on the expected losses.

Proposition 2. Suppose that $f(Q R)_{E L(\gamma,[z]])}=f\left(Q R_{\tau_{\bullet \rho}}\right)^{P o b(D \|[z])} f\left(Q R_{\tau_{\bullet \eta}}\right)^{P o b(\bar{D} \mid[z])}$, where $\operatorname{Pob}(D \mid[z])$ and $q$ are constant. Then, $f(Q R)_{E L(\gamma /[z])}$ is non-monotonic decreasing with respect to $f\left(Q R_{\tau_{\bullet \rho}}\right)$ and $f\left(Q R_{\tau_{\bullet \eta}}\right)(\bullet=\rho, \beta, \eta)$.

The proof of Proposition 2 is provided in Appendix 2.

In what follows, we explore the effect of the conditional possibility on the expected losses.

Proposition 3. Suppose that $t(Q R)_{E L(\gamma,[z])}=\left(1-\left(1-t\left(Q R_{\tau_{\bullet}}\right)^{q}\right)^{P o b(D[[z])}\left(1-t\left(Q R_{\tau_{\bullet}}\right)^{q}\right)^{P o b(\bar{D}[[z])}\right)^{1 / q}$, where $t\left(Q R_{\tau_{\bullet \rho}}\right), t\left(Q R_{\tau_{\bullet}}\right)(\bullet=\rho, \beta, \eta)$ and $q$ are constant. Then,

(1) If $t\left(Q R_{\tau_{\bullet \rho}}\right)=1$ or $t\left(Q R_{\tau_{v_{\eta}}}\right)=1, t(Q R)_{E L\left(\gamma_{*} \|[z]\right)}=1$.

(2) If $0 \leq t\left(Q R_{\tau_{\tau_{\eta}}}\right)<t\left(Q R_{\tau_{\sigma_{\rho}}}\right)<1, \quad t(Q R)_{E L\left(\gamma_{0} \|[z]\right)}$ is monotonic increasing with respect to $P$ ([D [ [ [ ] $]$ ).

(3) If $0 \leq t\left(Q R_{\tau_{\sigma_{\rho}}}\right) \leq t\left(Q R_{\tau_{\omega_{\eta}}}\right)<1, t(Q R)_{E L\left(\gamma_{\bullet} \mid[z]\right)}$ is non-monotonic increasing with respect to $\operatorname{Pob}(D \mid[z])$.

The proof of Proposition 3 is provided in Appendix 3.

Proposition 4. Suppose that $f(Q R)_{E L(\gamma,[z])}=f\left(Q R_{\tau_{\bullet \rho}}\right)^{P o b(D \mid[z])} f\left(Q R_{\tau_{\bullet \bullet}}\right)^{P o b(\bar{D}[z])}$, where $f\left(Q R_{\tau_{\bullet \rho}}\right)$ and $f\left(Q R_{\tau_{\bullet \eta}}\right)(\bullet=\rho, \beta, \eta)$ are constant. Then,

(1) If $f\left(Q R_{\tau_{\bullet \rho}}\right)=0$ or $f\left(Q R_{\tau_{\bullet_{\eta}}}\right)=0, f(Q R)_{E L\left(\gamma_{*} \|[z]\right)}=0$.

(2) If $0<f\left(Q R_{\tau_{\bullet_{\eta}}}\right)^{P o b(\bar{D} \mid[z])}<f\left(Q R_{\tau_{\bullet_{\rho}}}\right)^{P o b(D \mid[z])} \leq 1, f(Q R)_{E L\left(\gamma_{*} \|[z]\right)}$ is monotonic increasing with respect to $\operatorname{Pob}(D \mid[z])$.

(3) If $0<f\left(Q R_{\tau_{\omega_{\rho}}}\right)^{P o b(D[z])} \leq f\left(Q R_{\tau_{\omega_{\nu}}}\right)^{P o b(\bar{D} \mid[z])}<1, \quad f(Q R)_{E L(\gamma,[z])}$ is non-monotonic increasing with respect to $\operatorname{Pob}(D \mid[z])$.

The proof of Proposition 4 is provided in Appendix 4. 
In the context of the $q$-ROF environment, we can construct the following minimum-cost decision laws:

(T) If $E L\left(\gamma_{\rho} \mid[z]\right) \leq E L\left(\gamma_{\beta} \mid[z]\right)$ and $E L\left(\gamma_{\rho} \mid[z]\right) \leq E L\left(\gamma_{\eta} \mid[z]\right)$, determine $z \in \operatorname{Pos}(D)$;

(I) If $E L\left(\gamma_{\beta} \mid[z]\right) \leq E L\left(\gamma_{\rho} \mid[z]\right)$ and $E L\left(\gamma_{\beta} \mid[z]\right) \leq E L\left(\gamma_{\eta} \mid[z]\right)$, determine $z \in \operatorname{Bou}(D)$;

(F) If $E L\left(\gamma_{\eta} \mid[z]\right) \leq E L\left(\gamma_{\rho} \mid[z]\right)$ and $E L\left(\gamma_{\eta} \mid[z]\right) \leq E L\left(\gamma_{\beta} \mid[z]\right)$, determine $z \in N e g(D)$.

These TIF decision laws represent the 3WDs presented by Yao [57], with the positive law $\mathrm{T}$ representing the accept decision $(\mathrm{P})$, the boundary law (I) representing the non-commitment decision $(\mathrm{B})$, and the negative law $(\mathrm{F})$ representing the rejection decision $(\mathrm{N})$.

\section{The methods for obtaining 3WDs with the $q$-ROFDTRS model}

We propose a novel $q$-ROFDTRS model in Section 4. Regarding the TIF decision laws, the expected losses take the form of $q$-ROFNs. Under the circumstances, we are not able to judge them directly. Thus, we need to further analyse the TIF decision laws. In what follows, we propose two approaches to obtain 3 WDs. The first one is a comparative technique by utilizing the score and accuracy of $q$-ROFNs. The second one is a comparative technique by utilizing the possibility degree. Then, we provide the procedures of the proposed methods in detail.

\subsection{Approach 1: A comparative technique by utilizing the score and accuracy of $q$-ROFNs.}

A comparative technique for obtaining 3WDs based on Definition 5 is proposed here. First, by formulas (18)-(20), we express the expected losses as $E L\left(\gamma_{\rho} \mid[z]\right)=\left\langle t\left(Q R_{E L\left(\gamma_{\rho} \mid[z]\right)}\right), f\left(Q R_{E L\left(\gamma_{\rho} \mid[z]\right)}\right)\right\rangle$,

$$
\begin{aligned}
& E L\left(\gamma_{\beta} \mid[z]\right)=\left\langle t\left(Q R_{E L\left(\gamma_{\beta} \mid[z]\right)}\right), f\left(Q R_{E L\left(\gamma_{\beta} \mid[z]\right)}\right)\right\rangle \text { and } E L\left(\gamma_{\eta} \mid[z]\right)=\left\langle t\left(Q R_{E L\left(\gamma_{\eta} \mid[z]\right)}\right), f\left(Q R_{E L\left(\gamma_{\eta} \mid[z]\right)}\right)\right\rangle \text {, where } \\
& t(Q R)_{E L\left(\gamma_{\rho} \mid[z]\right)}=\left(1-\left(1-t\left(Q R_{\tau_{\rho \rho}}\right)^{q}\right)^{\operatorname{Pob}(D \mid[z])}\left(1-t\left(Q R_{\tau_{\rho \eta}}\right)^{q}\right)^{\operatorname{Pob}(\bar{D} \mid[z])}\right)^{1 / q}, \\
& f(Q R)_{E L\left(\gamma_{\rho} \|[z]\right)}=f\left(Q R_{\tau_{\rho \rho}}\right)^{\operatorname{Pob}(D \|[z])} f\left(Q R_{\tau_{\rho \eta}}\right)^{\operatorname{Pob}(\bar{D} \mid[z])}, \\
& t(Q R)_{E L\left(\gamma_{\beta} \|[z]\right)}=\left(1-\left(1-t\left(Q R_{\tau_{\beta \rho}}\right)^{q}\right)^{P o b(D \mid[z])}\left(1-t\left(Q R_{\tau_{\beta \eta}}\right)^{q}\right)^{P o b(\bar{D}[[z])}\right)^{1 / q}, \\
& f(Q R)_{E L\left(\gamma_{\beta} \mid[z]\right)}=f\left(Q R_{\tau_{\beta \rho}}\right)^{\operatorname{Pob}(D \mid[z])} f\left(Q R_{\tau_{\beta \eta}}\right)^{\operatorname{Pob}(\bar{D} \mid[z])}, \\
& t(Q R)_{E L\left(\gamma_{\eta} \mid[z]\right)}=\left(1-\left(1-t\left(Q R_{\tau_{\eta \rho}}\right)^{q}\right)^{\operatorname{Pob}(D \mid[z])}\left(1-t\left(Q R_{\tau_{\eta \eta}}\right)^{q}\right)^{\operatorname{Pob}(\bar{D} \mid[z])}\right)^{1 / q}, \\
& f(Q R)_{E L\left(\gamma_{\eta} \mid[z]\right)}=f\left(Q R_{\tau_{\eta \rho}}\right)^{\operatorname{Pob}(D \|[z])} f\left(Q R_{\tau_{\eta \eta}}\right)^{\operatorname{Pob}(\bar{D} \mid[z])} .
\end{aligned}
$$

Then, based on Definition 3, we can represent the score values of $E L\left(\gamma_{\rho} \mid[z]\right), E L\left(\gamma_{\beta} \mid[z]\right)$ and $E L\left(\gamma_{\eta} \mid[z]\right)$ as:

$$
\begin{aligned}
& S\left(E L\left(\gamma_{\rho} \mid[z]\right)\right)=t\left(Q R_{E L\left(\gamma_{\rho} \mid[z]\right)}\right)^{q}-f\left(Q R_{E L\left(\gamma_{\rho} \mid[z]\right)}\right)^{q}, \\
& S\left(E L\left(\gamma_{\beta} \mid[z]\right)\right)=t\left(Q R_{E L\left(\gamma_{\beta} \mid[z]\right)}\right)^{q}-f\left(Q R_{E L\left(\gamma_{\beta} \mid[z]\right)}\right)^{q},
\end{aligned}
$$




$$
\left.S\left(E L\left(\gamma_{\eta} \mid[z]\right]\right)\right)=t\left(Q R_{E L\left(\gamma_{\eta} \|[z]\right)}\right)^{q}-f\left(Q R_{E L\left(\gamma_{\eta}[[z]]\right.}\right)^{q} .
$$

In addition, based on Definition 4 , we can represent the accuracy values of $E L\left(\gamma_{\rho} \mid[z]\right)$, $E L\left(\gamma_{\beta} \mid[z]\right)$ and $E L\left(\gamma_{\eta} \mid[z]\right)$ as:

$$
\begin{aligned}
& H\left(E L\left(\gamma_{\rho} \mid[z]\right)\right)=t\left(Q R_{E L\left(\gamma_{\rho} \|[z]\right]}\right)^{q}+f\left(Q R_{E L\left(\gamma_{\rho} \|[z]\right]}\right)^{q}, \\
& H\left(E L\left(\gamma_{\beta} \mid[z]\right)\right)=t\left(Q R_{E L\left(\gamma_{\beta} \|[z]\right)}\right)^{q}+f\left(Q R_{E L\left(\gamma_{\beta} \|[z]\right)}\right)^{q}, \\
& H\left(E L\left(\gamma_{\eta} \mid[z]\right)\right)=t\left(Q R_{E L\left(\gamma_{\eta}[[z]]\right.}\right)^{q}+f\left(Q R_{E L\left(\gamma_{\eta}[[z]]\right.}\right)^{q}
\end{aligned}
$$

Thus, we can denote the TIF decision laws as follows:

$\left(\mathrm{T}^{1}\right)$ If $\left(S\left(E L\left(\gamma_{\rho} \mid[z]\right)\right)=S\left(E L\left(\gamma_{\beta} \mid[z]\right)\right) \wedge H\left(E L\left(\gamma_{\rho} \mid[z]\right)\right)<H\left(E L\left(\gamma_{\beta} \mid[z]\right)\right)\right)$

$$
\begin{aligned}
& \vee\left(S\left(E L\left(\gamma_{\rho} \mid[z]\right)\right)<S\left(E L\left(\gamma_{\beta} \mid[z]\right)\right)\right) \wedge\left(S\left(E L\left(\gamma_{\rho} \mid[z]\right)\right)<S\left(E L\left(\gamma_{\eta} \mid[z]\right)\right)\right) \\
& \left.\vee\left(S\left(E L\left(\gamma_{\rho} \mid[z]\right)\right)\right)=S\left(E L\left(\gamma_{\eta} \mid[z]\right)\right) \wedge H\left(E L\left(\gamma_{\rho} \mid[z]\right)\right)<H\left(E L\left(\gamma_{\eta} \mid[z]\right)\right)\right),
\end{aligned}
$$

determine $z \in \operatorname{Pos}(D)$;

$\left(\mathrm{I}^{1}\right)$ If $\left(S\left(E L\left(\gamma_{\beta} \mid[z]\right)\right)=S\left(E L\left(\gamma_{\rho} \mid[z]\right)\right) \wedge H\left(E L\left(\gamma_{\beta} \mid[z]\right)\right)<H\left(E L\left(\gamma_{\rho} \mid[z]\right)\right)\right)$

$$
\begin{aligned}
& \vee\left(S\left(E L\left(\gamma_{\beta} \mid[z]\right)\right)<S\left(E L\left(\gamma_{\rho} \mid[z]\right)\right)\right) \wedge\left(S\left(E L\left(\gamma_{\beta} \mid[z]\right)\right)<S\left(E L\left(\gamma_{\eta} \mid[z]\right)\right)\right) \\
& \vee\left(S\left(E L\left(\gamma_{\beta} \mid[z]\right)\right)=S\left(E L\left(\gamma_{\eta} \mid[z]\right)\right) \wedge H\left(E L\left(\gamma_{\beta} \mid[z]\right)\right)<H\left(E L\left(\gamma_{\eta} \mid[z]\right)\right)\right),
\end{aligned}
$$

determine $z \in \operatorname{Bou}(D)$;

$\left(\mathrm{F}^{1}\right)$ If $\left(S\left(E L\left(\gamma_{\eta} \mid[z]\right)\right)=S\left(E L\left(\gamma_{\rho} \mid[z]\right)\right) \wedge H\left(E L\left(\gamma_{\eta} \mid[z]\right)\right)<H\left(E L\left(\gamma_{\rho} \mid[z]\right)\right)\right)$

$$
\begin{aligned}
& \vee\left(S\left(E L\left(\gamma_{\eta} \mid[z]\right)\right)<S\left(E L\left(\gamma_{\rho} \mid[z]\right)\right)\right) \wedge\left(S\left(E L\left(\gamma_{\eta} \mid[z]\right)\right)<S\left(E L\left(\gamma_{\beta} \mid[z]\right)\right)\right) \\
& \left.\vee\left(S\left(E L\left(\gamma_{\eta} \mid[z]\right)\right)\right)=S\left(E L\left(\gamma_{\beta} \mid[z]\right)\right) \wedge H\left(E L\left(\gamma_{\eta} \mid[z]\right)\right)<H\left(E L\left(\gamma_{\beta} \mid[z]\right)\right)\right),
\end{aligned}
$$

determine $z \in \operatorname{Neg}(D)$.

Based on TIF $^{1}$ decision laws, we are now able to determine the corresponding decision law for $z$ directly.

\subsection{Approach 2: A comparative technique by utilizing possibility degrees.}

The comparative technique developed in Section 5.1 can be used to rank the different $q$-ROFNs. However, it cannot reflect the amount of the possibility degree of ranking different $q$-ROFNs. To offer more information to experts based on the possibility degree, we propose a possibility degree technique for ranking $q$-ROFNs by extending the possibility degree formula of IFSs [44]. The characteristic of this technique is that the comparing of $q$-ROFNs is transformed to the possibility degree of such a comparison, which is shown as follows:

Definition 6. Suppose $Q R_{1}=\left\langle t\left(Q R_{1}\right), f\left(Q R_{1}\right)\right\rangle$ and $Q R_{2}=\left\langle t\left(Q R_{2}\right), f\left(Q R_{2}\right)\right\rangle$ are any two $q$-ROFNs, and suppose $\varsigma\left(Q R_{1}\right)^{q}=1-\left(t\left(Q R_{1}\right)^{q}+f\left(Q R_{1}\right)^{q}\right)$ and $\varsigma\left(Q R_{2}\right)^{q}=1-\left(t\left(Q R_{2}\right)^{q}+f\left(Q R_{2}\right)^{q}\right)$ are not zero simultaneously. Then, the possibility degree of $q$-ROFNs is expressed as:

$$
p\left(Q R_{1}>Q R_{2}\right)=\frac{\max \left\{0, t\left(Q R_{1}\right)^{q}+\varsigma\left(Q R_{1}\right)^{q}-t\left(Q R_{2}\right)^{q}\right\}-\max \left\{0, t\left(Q R_{1}\right)^{q}-t\left(Q R_{1}\right)^{q}-\varsigma\left(Q R_{2}\right)^{q}\right\}}{\varsigma\left(Q R_{1}\right)^{q}+\varsigma\left(Q R_{2}\right)^{q}} .
$$


Definition 7. Suppose $Q R_{1}=\left\langle t\left(Q R_{1}\right), f\left(Q R_{1}\right)\right\rangle$ and $Q R_{2}=\left\langle t\left(Q R_{2}\right), f\left(Q R_{2}\right)\right\rangle$ are any two $q$-ROFNs, and suppose $\varsigma\left(Q R_{1}\right)^{q}=1-\left(t\left(Q R_{1}\right)^{q}+f\left(Q R_{1}\right)^{q}\right)$ and $\varsigma\left(Q R_{2}\right)^{q}=1-\left(t\left(Q R_{2}\right)^{q}+f\left(Q R_{2}\right)^{q}\right)$ are zero simultaneously. Then, the possibility degree of $q$-ROFNs is expressed as:

$$
p\left(Q R_{1}>Q R_{2}\right)= \begin{cases}1, & t\left(Q R_{1}\right)>t\left(Q R_{2}\right) ; \\ 0, & t\left(Q R_{1}\right)<t\left(Q R_{2}\right) ; \\ \frac{1}{2}, & t\left(Q R_{1}\right)=t\left(Q R_{2}\right) .\end{cases}
$$

It is easy to prove that the above definitions meet the properties as follows:

(1) $0 \leq p\left(Q R_{1}>Q R_{2}\right) \leq 1$;

(2) $p\left(Q R_{1}>Q R_{1}\right)=\frac{1}{2}$;

(3) $p\left(Q R_{1}>Q R_{2}\right)+p\left(Q R_{2}>Q R_{1}\right)=1$;

(4) $p\left(Q R_{1}>Q R_{2}\right)=1$ if $t\left(Q R_{1}\right)^{q} \geq t\left(Q R_{2}\right)^{q}+\varsigma\left(Q R_{2}\right)^{q}$;

(5) $p\left(Q R_{1}>Q R_{2}\right)=0$ if $t\left(Q R_{2}\right)^{q} \geq t\left(Q R_{1}\right)^{q}+\varsigma\left(Q R_{1}\right)^{q}$.

To rank $q$-ROFNs, $Q R_{k}=\left\langle t\left(Q R_{k}\right), f\left(Q R_{k}\right)\right\rangle(k=1,2, \ldots, m)$, we establish the matrix of the possibility degree as $P=\left(p_{k j}\right)_{m \times m}$, where $p_{k j}=p\left(Q R_{k}>Q R_{j}\right)(k, j=1,2, \ldots, m)$. Then, ranking vector $\xi=\left(\xi_{1}, \xi_{2}, \ldots, \xi_{m}\right)$ is calculated as:

$$
\xi_{k}=\frac{\left(\sum_{j=1}^{m} p_{k j}+\frac{m}{2}-1\right)}{m(m-1)}(k=1,2, \ldots, m) .
$$

Hence, the ranking order of $q$-ROFNs, $Q R_{k}=\left\langle t\left(Q R_{k}\right), f\left(Q R_{k}\right)\right\rangle(k=1,2, \ldots, m)$, can be obtained in decreasing order by the values of $\xi_{k}(k=1,2, \ldots, m)$. Based on the proposed ranking approach of different $q$-ROFNs, we now analyse the ranking order of $E L\left(\gamma_{\rho} \mid[z]\right), E L\left(\gamma_{\beta} \mid[z]\right)$ and $E L\left(\gamma_{\eta} \mid[z]\right)$ shown in formulas (18)-(20) and derive decision laws under the circumstance of $q$-ROFDTRSs. To begin with, the matrix of possibility degrees is established as listed in Table 3 .

Table 3. Matrix for the possibility degrees of $E L\left(\gamma_{\rho} \mid[z]\right), E L\left(\gamma_{\beta} \mid[z]\right)$ and $E L\left(\gamma_{\eta} \mid[z]\right)$

\begin{tabular}{c|c|c|c}
\hline$P$ & $E L\left(\gamma_{\rho} \mid[z]\right)$ & $E L\left(\gamma_{\beta} \mid[z]\right)$ & $E L\left(\gamma_{\eta} \mid[z]\right)$ \\
\hline$E L\left(\gamma_{\rho} \mid[z]\right)$ & $p_{11}=p\left(E L\left(\gamma_{\rho} \mid[z]\right)>E L\left(\gamma_{\rho} \mid[z]\right)\right)$ & $p_{12}=p\left(E L\left(\gamma_{\rho} \mid[z]\right)>E L\left(\gamma_{\beta} \mid[z]\right)\right)$ & $p_{13}=p\left(E L\left(\gamma_{\rho} \mid[z]\right)>E L\left(\gamma_{\eta} \mid[z]\right)\right)$ \\
\hline$E L\left(\gamma_{\beta} \mid[z]\right)$ & $p_{21}=p\left(E L\left(\gamma_{\beta} \mid[z]\right)>E L\left(\gamma_{\rho} \mid[z]\right)\right)$ & $p_{22}=p\left(E L\left(\gamma_{\beta} \mid[z]\right)>E L\left(\gamma_{\beta} \mid[z]\right)\right)$ & $p_{23}=p\left(E L\left(\gamma_{\beta} \mid[z]\right)>E L\left(\gamma_{\eta} \mid[z]\right)\right)$ \\
\hline$E L\left(\gamma_{\eta} \mid[z]\right)$ & $p_{31}=p\left(E L\left(\gamma_{\eta} \mid[z]\right)>E L\left(\gamma_{\rho} \mid[z]\right)\right)$ & $p_{32}=p\left(E L\left(\gamma_{\eta} \mid[z]\right)>E L\left(\gamma_{\beta} \mid[z]\right)\right)$ & $p_{33}=p\left(E L\left(\gamma_{\eta} \mid[z]\right)>E L\left(\gamma_{\eta} \mid[z]\right)\right)$ \\
\hline
\end{tabular}

From Table 3, the matrix for the possibility degrees of $E L\left(\gamma_{\rho} \mid[z]\right), E L\left(\gamma_{\beta} \mid[z]\right)$ and $E L\left(\gamma_{\eta} \mid[z]\right)$ is expressed as:

$$
P=\left(\begin{array}{lll}
p_{11} & p_{12} & p_{13} \\
p_{21} & p_{22} & p_{23} \\
p_{31} & p_{32} & p_{33}
\end{array}\right)
$$


Then, according to the properties of possibility degrees, we obtain: $0 \leq p_{k j} \leq 1, p_{k k}=\frac{1}{2}$, $p_{k j}+p_{j k}=1(k, j=1,2,3)$. The matrix for the possibility degrees of $E L\left(\gamma_{\rho} \mid[z]\right), E L\left(\gamma_{\beta} \mid[z]\right)$ and $E L\left(\gamma_{\eta} \mid[z]\right)$ is re-expressed as:

$$
P=\left(\begin{array}{ccc}
\frac{1}{2} & p_{12} & p_{13} \\
1-p_{12} & \frac{1}{2} & p_{23} \\
1-p_{13} & 1-p_{23} & \frac{1}{2}
\end{array}\right)
$$

Then, by (23), the ranking vector $\xi=\left(\xi_{1}, \xi_{2}, \xi_{3}\right)$ is expressed as:

$$
\xi=\left(\xi_{1}, \xi_{2}, \xi_{3}\right)=\left(\frac{1+p_{12}+p_{13}}{6}, \frac{2-p_{12}+p_{23}}{6}, \frac{3-p_{13}-p_{23}}{6}\right)
$$

Finally, by formula (24), we can denote the decision laws (T)-(F) in Section 4 as follows:

$\left(\mathrm{T}^{2}\right)$ If $\xi_{1} \leq \xi_{2}$ and $\xi_{1} \leq \xi_{3}$, determine $z \in \operatorname{Pos}(D)$;

(I $\left.{ }^{2}\right)$ If $\xi_{2} \leq \xi_{1}$ and $\xi_{2} \leq \xi_{3}$, determine $z \in \operatorname{Bou}(D)$;

$\left(\mathrm{F}^{2}\right)$ If $\xi_{3} \leq \xi_{1}$ and $\xi_{3} \leq \xi_{2}$, determine $z \in \operatorname{Neg}(D)$.

Based on $\mathrm{TIF}^{2}$ decision laws, we can determine the corresponding decision law for $z$ directly.

\subsection{The decision-making procedure of 3WDs}

Based on the aforementioned results, we further provide the decision-making procedure of 3WDs in the $q$-ROF setting using two comparative techniques, which includes the following detailed steps:

Step 1: Assessment of loss functions. In light of the real-word situation, the notion of $D$ and the alternatives $Z=\left\{z_{1}, z_{2}, \ldots, z_{m}\right\}$ are identified. Then, the loss functions of Table 2 are determined, namely, $Q R_{\tau_{\rho \rho}}=\left(t\left(Q R_{\tau_{\rho \rho}}\right), f\left(Q R_{\tau_{\rho \rho}}\right)\right), \quad Q R_{\tau_{\beta \rho}}=\left(t\left(Q R_{\tau_{\beta \rho}}\right), f\left(Q R_{\tau_{\beta \rho}}\right)\right), \quad Q R_{\tau_{p \rho}}=\left(t\left(Q R_{\tau_{p \rho}}\right), f\left(Q R_{\tau_{\eta \rho}}\right)\right)$, $Q R_{\tau_{\rho \eta}}=\left(t\left(Q R_{\tau_{\rho \eta}}\right), f\left(Q R_{\tau_{\rho \eta}}\right)\right), \quad Q R_{\tau_{\beta \eta}}=\left(t\left(Q R_{\tau_{\beta \eta}}\right), f\left(Q R_{\tau_{\beta \eta}}\right)\right)$ and $Q R_{\tau_{\eta \eta}}=\left(t\left(Q R_{\tau_{\eta \eta}}\right), f\left(Q R_{\tau_{\eta \eta}}\right)\right)$.

Step 2: Determination of conditional probability. Regarding the conditional probabilities $\operatorname{Pob}\left(D \mid z_{k}\right) \quad(k=1,2, \ldots, m)$, they are usually calculated in light of the experiences of experts or based on rough membership indicated by the nature of the information system [28].

Step 3: Computation of expected losses. The expected losses $E L\left(\gamma_{\rho} \mid z_{k}\right), E L\left(\gamma_{\beta} \mid z_{k}\right)$ and $E L\left(\gamma_{\eta} \mid z_{k}\right)$ for the alternative $z_{k}(k=1,2, \ldots, m)$ under a provided value of $\operatorname{Pob}\left(D \mid z_{k}\right)$ are computed successively using formulas (18)-(20).

Step 4: Comparison of expected losses. If the first comparative technique is used to compare the expected losses, the following three sub-steps are involved. First, the scores of $\operatorname{EL}\left(\gamma_{\rho} \mid z_{k}\right)$, $E L\left(\gamma_{\beta} \mid z_{k}\right)$ and $E L\left(\gamma_{\eta} \mid z_{k}\right)$ are computed based on Definition 3. Then, the accuracies of $E L\left(\gamma_{\rho} \mid z_{k}\right)$, 
$E L\left(\gamma_{\beta} \mid z_{k}\right)$ and $E L\left(\gamma_{\eta} \mid z_{k}\right)$ are computed based on Definition 4. Finally, decision laws TIF $^{1}$ are used to determine the corresponding decision law for each alternative $z_{k}(k=1,2, \ldots, m)$. If the second comparative technique is used, the following three sub-steps are involved. First, the matrices $P_{k}$ for the possibility degree for each alternative $z_{k}(k=1,2, \ldots, m)$ are constructed using formulas (21) and (22). Then, the ranking vectors $\xi_{k}$ of the expected losses for each alternative $z_{k}(k=1,2, \ldots, m)$ are computed. Finally, the decision laws $\mathrm{TIF}^{2}$ are used to determine the relevant decision law for each alternative.

We can finally derive the decision result for each alternative using Steps 1-4. Steps 1-2 denote the preparation phases. The time complexity of Step 3 is linear. For Step 4, the time complexities of the first and second comparative techniques are linear. Therefore, the time complexities of our developed approaches are linear. For clarity, the decision-making procedure of our proposed approaches is depicted in Figure 2.

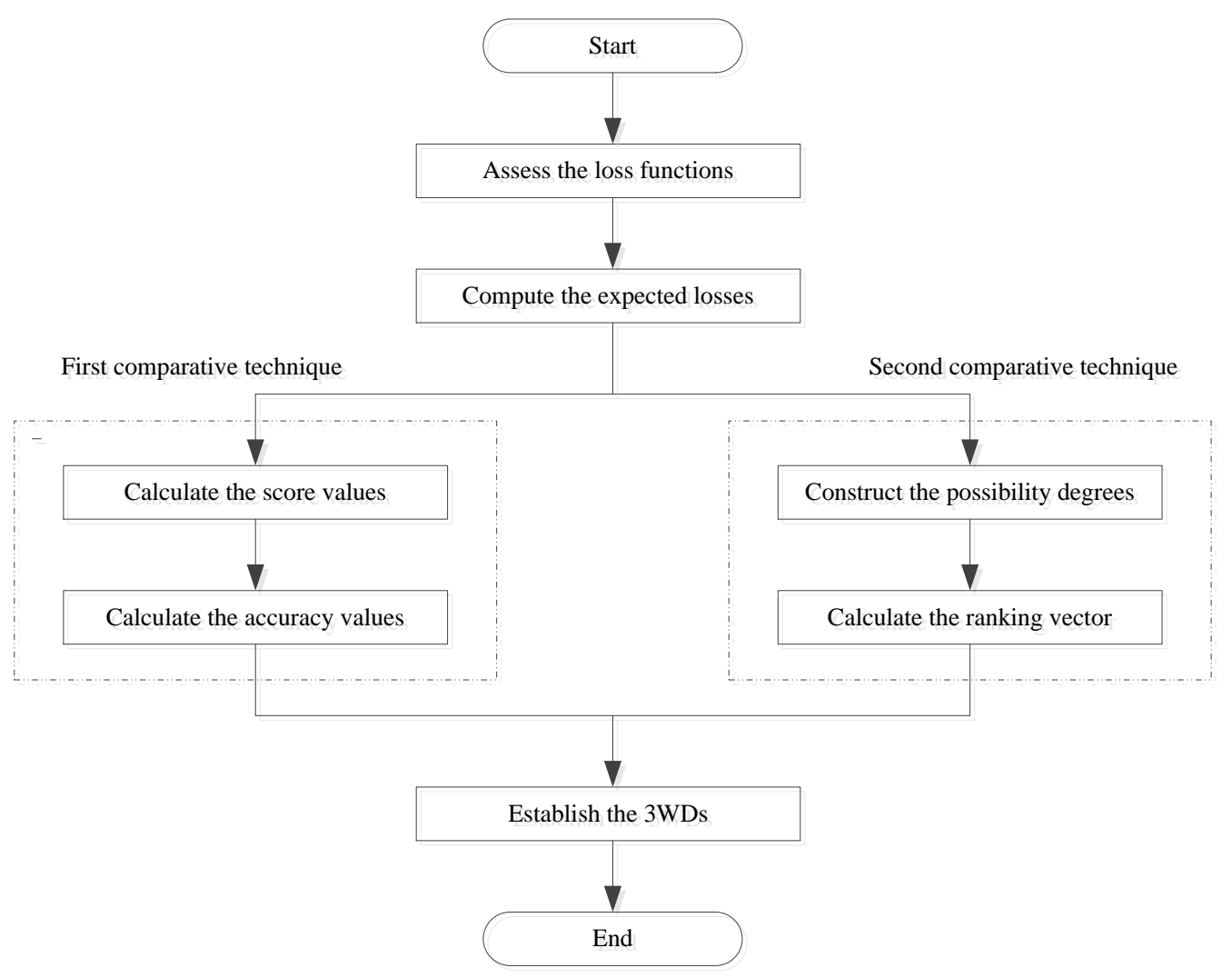

Figure 2. Decision procedure of the proposed approaches.

\section{Practical cases}

In this part, an illustrative instance concerning stock investment is utilized to verify the procedure of the created methods. Then, to demonstrate the viability and advantages of the proposed methods, we conduct a comparative analysis with Liang and Liu's method [24] and Liang et al.'s method [31].

\subsection{The decision-making procedure of the proposed methods}

Example 1. With respect to the selection decisions of stock investment, suppose $\Theta=\{D, \bar{D}\}$ is 
the collection of states expressing whether the stock $z$ has a good market prospect or not. Suppose $\Lambda=\left\{\gamma_{\rho}, \gamma_{\beta}, \gamma_{\eta}\right\}$ is the collection of actions for $z$, where $\gamma_{\rho}, \gamma_{\beta}$ and $\gamma_{\eta}$ represent investment, further investigation, and investment rejection, respectively. Suppose $\operatorname{Pob}(D \mid[z])$ is the conditional probability denoting the probability of $z$ having a good market prospect. From Table 2, these loss functions are denoted as: $Q R_{\tau_{\rho \rho}}=\left(t\left(Q R_{\tau_{\rho \rho}}\right), f\left(Q R_{\tau_{\rho \rho}}\right)\right), \quad Q R_{\tau_{\beta \rho}}=\left(t\left(Q R_{\tau_{\beta \rho}}\right), f\left(Q R_{\tau_{\beta \rho}}\right)\right)$, $Q R_{\tau_{\eta p}}=\left(t\left(Q R_{\tau_{\eta p}}\right), f\left(Q R_{\tau_{\eta \rho}}\right)\right), \quad Q R_{\tau_{\rho \eta}}=\left(t\left(Q R_{\tau_{\rho \eta}}\right), f\left(Q R_{\tau_{\rho \eta}}\right)\right), \quad Q R_{\tau_{\beta \eta}}=\left(t\left(Q R_{\tau_{\beta \eta}}\right), f\left(Q R_{\tau_{\beta \eta}}\right)\right) \quad$ and $Q R_{\tau_{\eta \eta}}=\left(t\left(Q R_{\tau_{\eta \eta}}\right), f\left(Q R_{\tau_{\eta \eta}}\right)\right)$. In this situation, $Q R_{\tau_{\rho \rho}}, Q R_{\tau_{\beta \rho}}$ and $Q R_{\tau_{\eta \rho}}$ represent the losses with $q$-ROFNs incurred for adopting measures of investment, further investigation, and investment rejection, respectively, while $z$ has a good market prospect. Analogously, $Q R_{\tau_{\rho \eta}}, Q R_{\tau_{\beta \eta}}$ and $Q R_{\tau_{\eta \eta}}$ represent the losses with $q$-ROFNs incurred for investing, further investigating, and rejecting investment, respectively, while $z$ has a bad market prospect. By the decision-making methods with $q$-ROFDTRSs, we explain the decision process for the stock investment as follows:

[Step 1] Suppose that there are six stocks expressed as $Z=\left\{z_{1}, z_{2}, \ldots, z_{6}\right\}$, and then five experts are invited from a consulting company, who have rich experience in the assessment of stock investment, to provide the loss values. For instance, when the first expert evaluates the information on loss function $Q R_{\tau_{\rho \rho}}=\left(t\left(Q R_{\tau_{\rho \rho}}\right), f\left(Q R_{\tau_{\rho \rho}}\right)\right)$, he/she thinks its membership degree and non-membership degree are 0.2 and 0.7 , respectively, so he/she thinks its value is $(0.2,0.7)$. In this way, the other experts think its values are $(0.1,0.6),(0.1,0.8),(0,0.4)$ and $(0.1,0.6)$. Therefore, the loss value $Q R_{\tau_{\rho \rho}}=(0.1,0.6)$ is derived using the average operator. Analogously, all the loss values are derived, which are shown as follows:

$$
\begin{aligned}
& Q R_{\tau_{\rho \rho}}=(0.1,0.6), Q R_{\tau_{\beta \rho}}=(0.7,0.2), Q R_{\tau_{\eta \rho}}=(0.9,0.1), \\
& Q R_{\tau_{\rho \eta}}=(0.8,0.1), Q R_{\tau_{\beta \eta}}=(0.1,0.6), Q R_{\tau_{\eta \eta}}=(0.01,0.75) .
\end{aligned}
$$

[Step 2] In this example, the conditional probabilities for six stocks provided by experts are shown in Table 4.

Table 4. Conditional probability for six stocks $Z$ having a good market prospect.

\begin{tabular}{c|c|c|c|c|c|c}
\hline$Z$ & $z_{1}$ & $z_{2}$ & $z_{3}$ & $z_{4}$ & $z_{5}$ & $z_{6}$ \\
\hline$P o b(D \mid[z])$ & 0.05 & 0.49 & 0.32 & 0.21 & 0.72 & 0.80 \\
\hline
\end{tabular}

[Step 3] Applying (18)-(20), we compute the expected losses values for each stock $z_{k}(k=1,2, \ldots, 6)$ as listed in Table 5 (without loss of generality, we let parameter $q=4$ ).

Table 5. Expected losses for each stock

\begin{tabular}{c|c|c|c}
\hline$Z$ & $E L\left(\gamma_{\rho} \mid[z]\right)$ & $E L\left(\gamma_{\beta} \mid[z]\right)$ & $E L\left(\gamma_{\eta} \mid[z]\right)$ \\
\hline$z_{1}$ & $(0.7922,0.1094)$ & $(0.3423,0.5679)$ & $(0.4755,0.6781)$ \\
\hline$z_{2}$ & $(0.6968,0.2406)$ & $(0.5957,0.3502)$ & $(0.7989,0.2794)$ \\
\hline$z_{3}$ & $(0.7408,0.1774)$ & $(0.5386,0.4222)$ & $(0.7334,0.3936)$ \\
\hline$z_{4}$ & $(0.7639,0.1457)$ & $(0.4867,0.4764)$ & $(0.6694,0.4912)$ \\
\hline$z_{5}$ & $(0.6087,0.3633)$ & $(0.6508,0.2720)$ & $(0.8558,0.1758)$ \\
\hline$z_{6}$ & $(0.5625,0.4193)$ & $(0.6664,0.2491)$ & $(0.8705,0.1496)$ \\
\hline
\end{tabular}


[Step 4] By utilizing the expected losses listed in Table 5 and the two methods developed in Section 5, we further determine the decision result for each stock. We first apply the first comparative technique by utilizing score functions and accuracy functions of $q$-ROFNs to obtain 3 WDs. According to Definitions 3 and 4 , we calculate score values and accuracy values of $E L\left(\gamma_{\rho} \mid z_{k}\right), E L\left(\gamma_{\beta} \mid z_{k}\right)$ and $E L\left(\gamma_{\eta} \mid z_{k}\right)$ for each stock $z_{k}(k=1,2, \ldots, 6)$, which are depicted in Figures 3 and 4 , respectively.

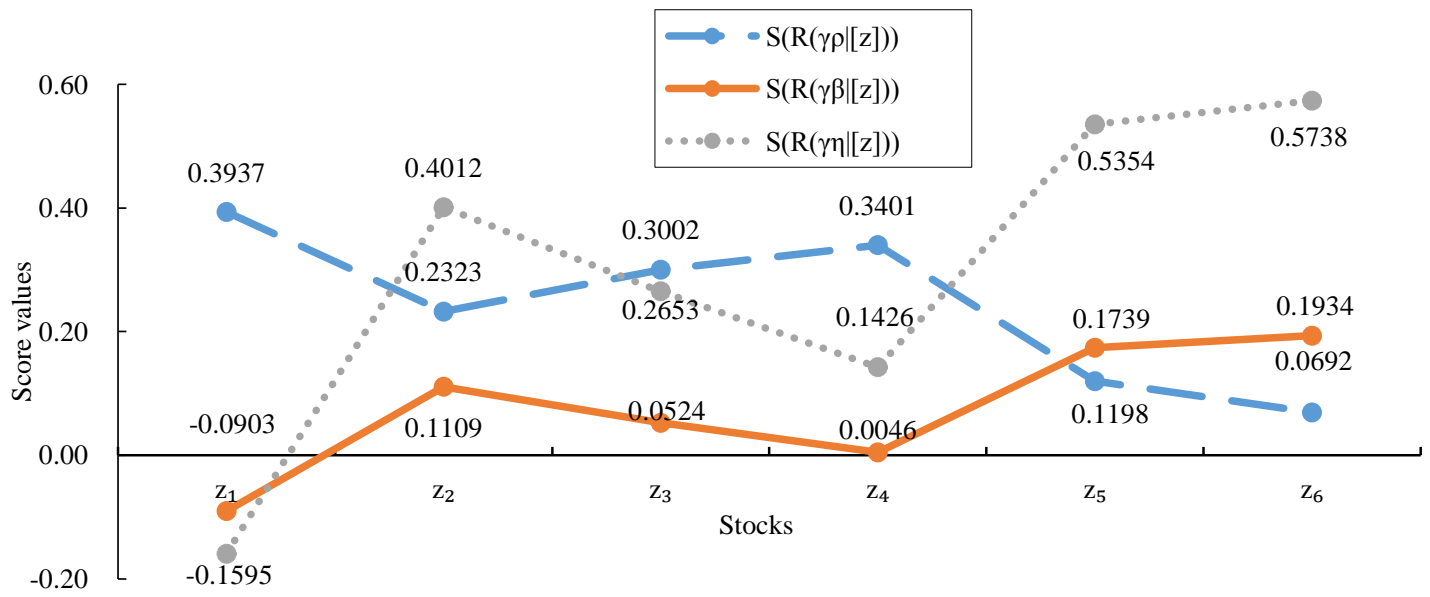

Figure 3. Score values of the related losses for each stock

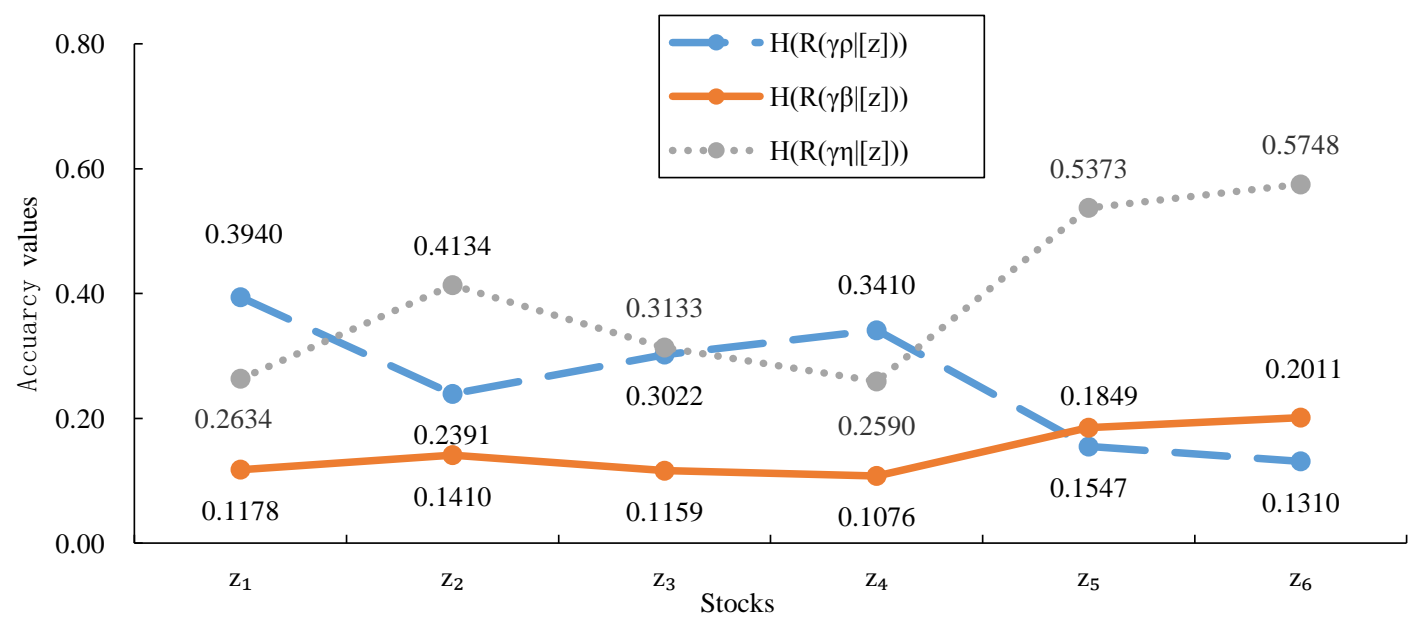

Figure 4. Accuracy values of the related losses for each stock

Based on TIF $^{1}$ decision laws, we are able to determine the relevant decision laws for the stocks, which are shown in Table 6.

Table 6. Decision results of the stocks with the comparative technique by the score function and the accuracy function of $q$-ROFNs

\begin{tabular}{c|c|c|c|c|c|c}
\hline$Z$ & $z_{1}$ & $z_{2}$ & $z_{3}$ & $z_{4}$ & $z_{5}$ & $z_{6}$ \\
\hline The investment judgement & $\operatorname{Neg}(D)$ & $\operatorname{Bou}(D)$ & $\operatorname{Bou}(D)$ & $\operatorname{Bou}(D)$ & $\operatorname{Pos}(D)$ & $\operatorname{Pos}(D)$ \\
\hline
\end{tabular}

From Table 6, we can obtain that $z_{1}$ should not be invested, while $z_{5}$ and $z_{6}$ need to be selected for investment immediately; additionally, $z_{2}, z_{3}$ and $z_{4}$ should be further investigated.

Now, we apply the second comparative technique developed in Section 5 by utilizing the 
possibility degree to obtain 3WDs. By formulas (21) and (22), we construct the matrices $P_{k}$ of possibility degrees for each stock $z_{k}(k=1,2, \ldots, 6)$ :

$$
\begin{aligned}
& P_{1}=\left(\begin{array}{lll}
0.5000 & 0.6626 & 0.7060 \\
0.3374 & 0.5000 & 0.5214 \\
0.2940 & 0.4786 & 0.5000
\end{array}\right), P_{2}=\left(\begin{array}{lll}
0.5000 & 0.5375 & 0.4374 \\
0.4625 & 0.5000 & 0.3996 \\
0.5626 & 0.6004 & 0.5000
\end{array}\right), P_{3}=\left(\begin{array}{lll}
0.5000 & 0.5783 & 0.5126 \\
0.4217 & 0.5000 & 0.4322 \\
0.4874 & 0.5678 & 0.5000
\end{array}\right), \\
& P_{4}=\left(\begin{array}{lll}
0.5000 & 0.6081 & 0.5705 \\
0.3919 & 0.5000 & 0.4578 \\
0.4295 & 0.5422 & 0.5000
\end{array}\right), P_{5}=\left(\begin{array}{lll}
0.5000 & 0.4837 & 0.3412 \\
0.5163 & 0.5000 & 0.3586 \\
0.6588 & 0.6414 & 0.5000
\end{array}\right), P_{6}=\left(\begin{array}{lll}
0.5000 & 0.4628 & 0.3051 \\
0.5372 & 0.5000 & 0.3446 \\
0.6949 & 0.6554 & 0.5000
\end{array}\right) \text {. }
\end{aligned}
$$

Then, by formula (24), we calculate the ranking vectors $\xi_{k}=\left(\delta_{k 1}, \delta_{k 2}, \delta_{k 3}\right)$ of the expected losses for each stock $z_{k}(k=1,2, \ldots, 6)$, which are depicted in Figure 5.

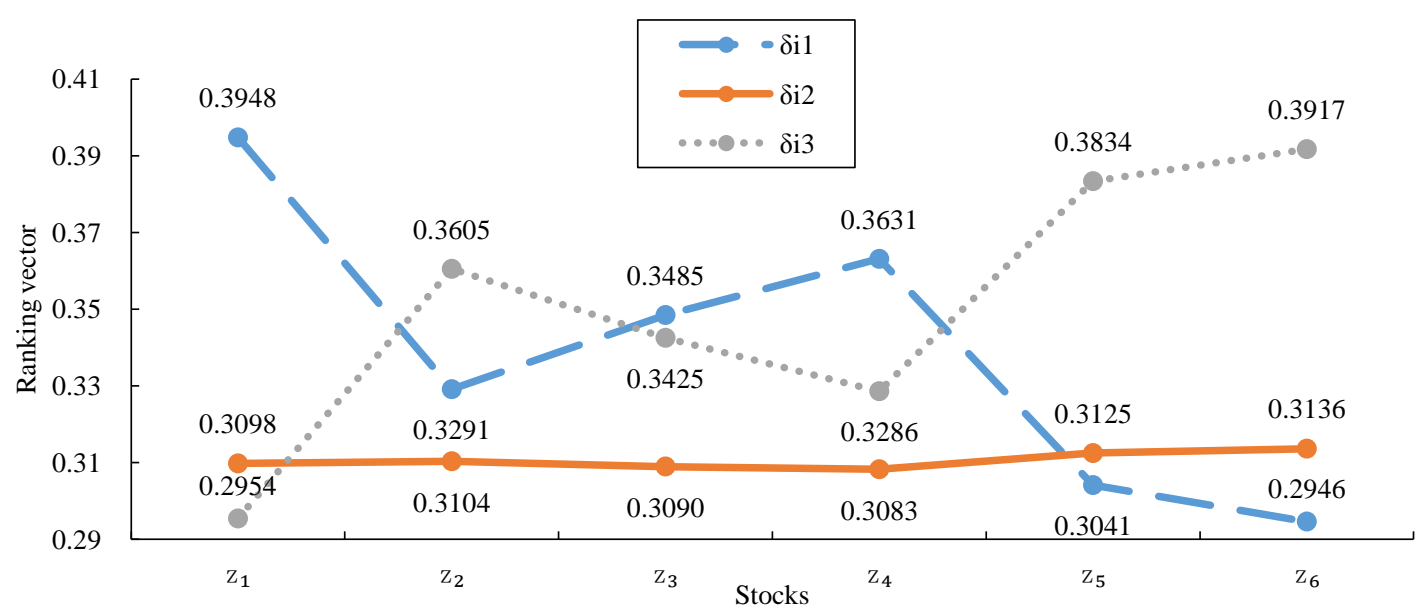

Figure 5. Ranking vector of the related losses for each stock

From Figures 3 and 5, we can observe that $\delta_{k 1}, \delta_{k 2}$ and $\delta_{k 3}$ follow a similar pattern to score values $S\left(R L\left(\gamma_{\rho} \mid[z]\right)\right), S\left(R L\left(\gamma_{\beta} \mid[z]\right)\right)$ and $S\left(R L\left(\gamma_{\eta} \mid[z]\right)\right)$, respectively.

Based on $\mathrm{TIF}^{2}$ decision laws, we are able to determine the relevant investment decision laws for the stocks, which are shown in Table 7.

Table 7. Decision results of the stocks with the second method by possibility degrees

\begin{tabular}{c|c|c|c|c|c|c}
\hline$Z$ & $z_{1}$ & $z_{2}$ & $z_{3}$ & $z_{4}$ & $z_{5}$ & $z_{6}$ \\
\hline The investment judgement & $\operatorname{Neg}(D)$ & $\operatorname{Bou}(D)$ & $\operatorname{Bou}(D)$ & $\operatorname{Bou}(D)$ & $\operatorname{Pos}(D)$ & $\operatorname{Pos}(D)$ \\
\hline
\end{tabular}

From Table 7, we can make a further judgement that $z_{1}$ should not be invested, while $z_{5}$ and $z_{6}$ need to be selected for investment immediately; additionally, $z_{2}, \quad z_{3}$ and $z_{4}$ should be investigated.

In this application example, we can make the investment decision of each stock by utilizing the two approaches developed in Section 5 as shown in Tables 6 and 7, which draw the same conclusion: $z_{1} \in \operatorname{Neg}(D), z_{2}, z_{3}, z_{4} \in \operatorname{Bou}(D)$ and $z_{5}, z_{6} \in \operatorname{Pos}(D)$.

Despite that these two ranking approaches achieve the same results, there exist some differences 
between these two methods. With respect to the complexity of computation, the first method is relatively simpler to perform. As computer technology develops rapidly, it could assist the second method and enable us to endure its complexity of calculation. However, it is worth pointing out that the second method has a prominent advantage because the possibility degrees between any adjacent $q$-ROFNs for the ranking order could be derived from the matrix of possibility degrees. For instance, for the stock $z_{1}$, by matrix $P_{1}$, we have: $E L\left(\gamma_{\rho} \mid\left[z_{1}\right]\right)>_{0.6626} E L\left(\gamma_{\beta} \mid\left[z_{1}\right]\right)>_{0.5214} E L\left(\gamma_{\eta} \mid\left[z_{1}\right]\right)$. In other words, the possibility degree of $E L\left(\gamma_{\rho} \mid\left[z_{1}\right]\right)>E L\left(\gamma_{\beta} \mid\left[z_{1}\right]\right)$ equals 0.6626 , and the possibility degree of $E L\left(\gamma_{\beta} \mid\left[z_{1}\right]\right)>E L\left(\gamma_{\eta} \mid\left[z_{1}\right]\right)$ equals 0.5214 . Clearly, since the second method by utilizing possibility degree offers more information regarding the comparison of $q$-ROFNs, we are more sure of $E L\left(\gamma_{\rho} \mid\left[z_{1}\right]\right)>E L\left(\gamma_{\beta} \mid\left[z_{1}\right]\right)$ than $E L\left(\gamma_{\beta} \mid\left[z_{1}\right]\right)>E L\left(\gamma_{\eta} \mid\left[z_{1}\right]\right)$. In some actual decision-making problems, this additional information might help experts to make reasonable decisions. Thus, when experts want to take advantage of the additional information for the comparison of $q$-ROFNs and endure a long calculation time, the second ranking method is the optimal choice. Otherwise, the first method is the best choice.

\subsection{Comparison with the existing methods}

\section{(1) Demonstrating the viability of the proposed methods}

To demonstrate the viability of our proposed methods, we compare the experimental outcomes of the created methods with the ones of Liang and Liu's method [24] and Liang et al.'s method [31]. Since we can determine the decision results only by the score values $S\left(R L\left(\gamma_{\rho} \mid\left[z_{k}\right]\right)\right), S\left(R L\left(\gamma_{\beta} \mid\left[z_{k}\right]\right)\right)$ and $S\left(R L\left(\gamma_{\eta} \mid\left[z_{k}\right]\right)\right)$ for each stock $z_{k}(k=1,2, \ldots, 6)$ in this example, the accuracy values are omitted here. The computation outcomes of Example 1 for different approaches are shown in Table 8 and Figure 6.

Table 8. Decision result of different approaches.

\begin{tabular}{|c|c|c|c|c|}
\hline Methods & Score values & $\operatorname{Pos}(D)$ & $\operatorname{Bou}(D)$ & $\operatorname{Neg}(D)$ \\
\hline $\begin{array}{l}\text { Liang and Liu's } \\
\text { method [24] }\end{array}$ & $\begin{array}{l}S\left(E L\left(\gamma_{\rho} \mid\left[z_{1}\right]\right)\right)=0.6750, S\left(E L\left(\gamma_{\beta} \mid\left[z_{1}\right]\right)\right)=-0.4198, S\left(E L\left(\gamma_{\eta} \mid\left[z_{1}\right]\right)\right)=-0.5609 \\
S\left(E L\left(\gamma_{\rho} \mid\left[z_{2}\right]\right)\right)=0.3415, S\left(E L\left(\gamma_{\beta} \mid\left[z_{2}\right]\right)\right)=0.1244, S\left(E L\left(\gamma_{\eta} \mid\left[z_{2}\right]\right)\right)=0.3986 \\
S\left(E L\left(\gamma_{\rho} \mid\left[z_{3}\right]\right)\right)=0.4989, S\left(E L\left(\gamma_{\beta} \mid\left[z_{3}\right]\right)\right)=-0.0554, S\left(E L\left(\gamma_{\eta} \mid\left[z_{3}\right]\right)\right)=0.1310 \\
S\left(E L\left(\gamma_{\rho} \mid\left[z_{4}\right]\right)\right)=0.5800, S\left(E L\left(\gamma_{\beta} \mid\left[z_{4}\right]\right)\right)=-0.1910, S\left(E L\left(\gamma_{\eta} \mid\left[z_{4}\right]\right)\right)=-0.1030 \\
S\left(E L\left(\gamma_{\rho} \mid\left[z_{5}\right]\right)\right)=0.0460, S\left(E L\left(\gamma_{\beta} \mid\left[z_{5}\right]\right)\right)=0.3199, S\left(E L\left(\gamma_{\eta} \mid\left[z_{5}\right]\right)\right)=0.6342 \\
S\left(E L\left(\gamma_{\rho} \mid\left[z_{6}\right]\right)\right)=-0.0855, S\left(E L\left(\gamma_{\beta} \mid\left[z_{6}\right]\right)\right)=0.3771, S\left(E L\left(\gamma_{\eta} \mid\left[z_{6}\right]\right)\right)=0.6922\end{array}$ & $\left\{z_{5}, z_{6}\right\}$ & $\left\{z_{2}, z_{3}, z_{4}\right\}$ & $\left\{z_{1}\right\}$ \\
\hline $\begin{array}{l}\text { Liang et al.'s } \\
\text { method [31] }\end{array}$ & $\begin{array}{l}S\left(E L\left(r_{\rho} \mid\left[z_{1}\right]\right)\right)=0.6094, S\left(E L\left(r_{\beta} \mid\left[z_{1}\right]\right)\right)=-0.2803, S\left(E L\left(r_{\eta} \mid\left[z_{1}\right]\right)\right)=-0.3801 \\
S\left(E L\left(r_{\rho} \mid\left[z_{2}\right]\right)\right)=0.3511, S\left(E L\left(r_{\beta} \mid\left[z_{2}\right]\right)\right)=0.1620, S\left(E L\left(r_{\eta} \mid\left[z_{2}\right]\right)\right)=0.4787 \\
S\left(E L\left(r_{\rho} \mid\left[z_{3}\right]\right)\right)=0.4709, S\left(E L\left(r_{\beta} \mid\left[z_{3}\right]\right)\right)=0.0211, S\left(E L\left(r_{\eta} \mid\left[z_{3}\right]\right)\right)=0.2574 \\
S\left(E L\left(r_{\rho} \mid\left[z_{4}\right]\right)\right)=0.5336, S\left(E L\left(r_{\beta} \mid\left[z_{4}\right]\right)\right)=-0.0882, S\left(E L\left(r_{\eta} \mid\left[z_{4}\right]\right)\right)=0.0532 \\
S\left(E L\left(r_{\rho} \mid\left[z_{5}\right]\right)\right)=0.1222, S\left(E L\left(r_{\beta} \mid\left[z_{5}\right]\right)\right)=0.3119, S\left(E L\left(r_{\eta} \mid\left[z_{5}\right]\right)\right)=0.6666 \\
S\left(E L\left(r_{\rho} \mid\left[z_{6}\right]\right)\right)=0.0155, S\left(E L\left(r_{\beta} \mid\left[z_{6}\right]\right)\right)=0.3556, S\left(E L\left(r_{\eta} \mid\left[z_{6}\right]\right)\right)=0.7128\end{array}$ & $\left\{z_{5}, z_{6}\right\}$ & $\left\{z_{2}, z_{3}, z_{4}\right\}$ & $\left\{z_{1}\right\}$ \\
\hline $\begin{array}{c}\text { Our proposed } \\
\text { methods }\end{array}$ & $\begin{array}{l}S\left(E L\left(\gamma_{\rho} \mid\left[z_{1}\right]\right)\right)=0.3937, S\left(E L\left(\gamma_{\beta} \mid\left[z_{1}\right]\right)\right)=-0.0903, S\left(E L\left(\gamma_{\eta} \mid\left[z_{1}\right]\right)\right)=-0.1595 \\
S\left(E L\left(\gamma_{\rho} \mid\left[z_{2}\right]\right)\right)=0.2323, S\left(E L\left(\gamma_{\beta} \mid\left[z_{2}\right]\right)\right)=0.1109, S\left(E L\left(\gamma_{\eta} \mid\left[z_{2}\right]\right)\right)=0.4012 \\
S\left(E L\left(\gamma_{\rho} \mid\left[z_{3}\right]\right)\right)=0.3002, S\left(E L\left(\gamma_{\beta} \mid\left[z_{3}\right]\right)\right)=0.0524, S\left(E L\left(\gamma_{\eta} \mid\left[z_{3}\right]\right)\right)=0.2653 \\
S\left(E L\left(\gamma_{\rho} \mid\left[z_{4}\right]\right)\right)=0.3401, S\left(E L\left(\gamma_{\beta} \mid\left[z_{4}\right]\right)\right)=0.0046, S\left(E L\left(\gamma_{\eta} \mid\left[z_{4}\right]\right)\right)=0.1426 ; \\
S\left(E L\left(\gamma_{\rho} \mid\left[z_{5}\right]\right)\right)=0.1198, S\left(E L\left(\gamma_{\beta} \mid\left[z_{5}\right]\right)\right)=0.1739, S\left(E L\left(\gamma_{\eta} \mid\left[z_{5}\right]\right)\right)=0.5354 \\
S\left(E L\left(\gamma_{\rho} \mid\left[z_{6}\right]\right)\right)=0.0692, S\left(E L\left(\gamma_{\beta} \mid\left[z_{6}\right]\right)\right)=0.1934, S\left(E L\left(\gamma_{\eta} \mid\left[z_{6}\right]\right)\right)=0.5738\end{array}$ & $\left\{z_{5}, z_{6}\right\}$ & $\left\{z_{2}, z_{3}, z_{4}\right\}$ & $\left\{z_{1}\right\}$ \\
\hline
\end{tabular}




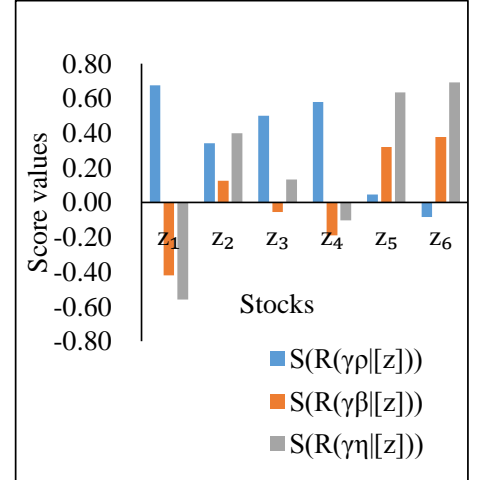

(1) Liang and Liu's method [24]

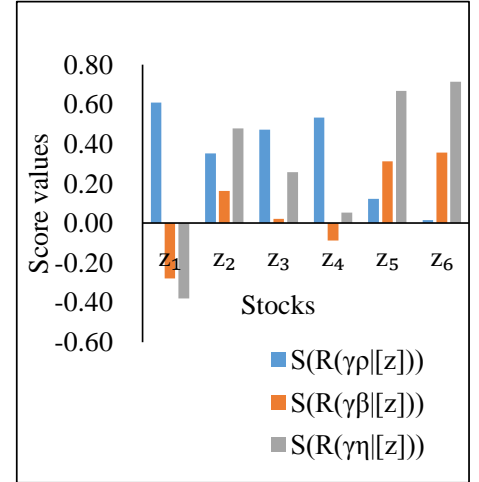

(2) Liang et al.'s method [31]

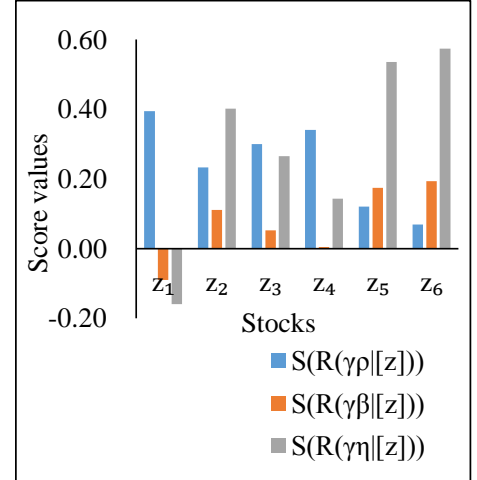

(3) Our proposed method

Figure 6. Decision result of different approaches.

From Table 8 and Figure 6, we find that Liang and Liu's method [24], Liang et al.'s method [31] and our proposed methods obtain the same decision result: $z_{1} \in N e g(D), z_{2}, z_{3}, z_{4} \in \operatorname{Bou}(D)$ and $z_{5}, z_{6} \in \operatorname{Pos}(D)$. Thus, the proposed methods are effective and viable.

\section{(2) Verifying the advantages of the proposed methods}

In the above part, the viability of the proposed methods has been demonstrated. However, since the decision outcomes obtained from our proposed methods, Liang and Liu's method [24] and Liang et al.'s method [31] coincide with one another, the superiority of the proposed methods cannot be reflected. In this part, to illustrate the advantages of the proposed methods, we conduct a contrastive analysis by continuously changing several loss values in Example 1. For instance, the loss value $Q R_{\tau_{\beta p}}$ is changed from $Q R_{\tau_{\beta \rho}}=(0.7,0.2)$ to $Q R_{\tau_{\beta \rho}}=(0.7,0.35)$. In this case, the decision outcomes for the different methods are listed in Table 9.

Table 9. Decision results of different approaches.

\begin{tabular}{l|c|c|c}
\hline \multicolumn{1}{c|}{ Methods } & $\operatorname{Pos}(D)$ & $\operatorname{Bou}(D)$ & Neg $(D)$ \\
\hline Liang and Liu's method [24] & Cannot obtain the decision & Cannot obtain the decision & Cannot obtain the decision \\
& result & result & result \\
\hline Liang et al.'s method [31] & $\left\{z_{5}, z_{6}\right\}$ & $\left\{z_{2}, z_{3}, z_{4}\right\}$ & $\left\{z_{1}\right\}$ \\
\hline Our proposed methods & $\left\{z_{5}, z_{6}\right\}$ & $\left\{z_{2}, z_{3}, z_{4}\right\}$ & $\left\{z_{1}\right\}$ \\
\hline
\end{tabular}

From Table 9, we can see that Liang et al.'s method [31] and our proposed methods obtain the same decision result, that is, $z_{1} \in \operatorname{Neg}(D), z_{2}, z_{3}, z_{4} \in \operatorname{Bou}(D)$ and $z_{5}, z_{6} \in \operatorname{Pos}(D)$, whereas Liang and Liu's method [24] has the drawback that it cannot deal with the modified application example. Indeed, Liang and Liu's method [24] can only handle evaluating loss values denoted by IFNs, where the $\mathrm{MD}, t\left(Q R_{\tau_{\bullet}}\right)$ and the NMD, $f\left(Q R_{\tau_{\bullet}}\right)$ of each loss value $Q R_{\tau_{\bullet}}(\bullet=\rho, \beta, \eta ; \circ=\rho, \eta)$ need to satisfy the condition that $0 \leq t\left(Q R_{\tau_{\bullet}}\right)+f\left(Q R_{\tau_{\bullet}}\right) \leq 1$. However, the adjusted loss value $Q R_{\tau_{\beta \rho}}=(0.7,0.35)$ is not an IFN because $0.7+0.35>1$. Thus, Liang and Liu's method [24] cannot obtain a decision result.

In addition, Liang et al.'s method [31] is developed by utilizing PFNs, where the MD, $t\left(Q R_{\tau_{*}}\right)$ 
and the NMD, $f\left(Q R_{\tau_{\bullet}}\right)$ of each loss value $Q R_{\tau_{\bullet}}(\bullet=\rho, \beta, \eta ; \circ=\rho, \eta)$ need to satisfy the condition that $t\left(Q R_{\tau_{*}}\right)^{2}+f\left(Q R_{\tau_{\tau_{0}}}\right)^{2} \leq 1$. Meanwhile, the adjusted loss value $Q R_{\tau_{\beta \rho}}=(0.7,0.35)$ is a PFN because $0.7^{2}+0.35^{2}<1$. Thus, Liang et al.'s method [31] can derive decision results. Clearly, PFNs are superior to the IFNs because they can depict estimating loss values more completely. However, Liang et al.'s method [31] still has two shortcomings compared with our proposed methods. First, the scope of information described by PFNs is still restricted. For instance, when the estimating value $Q R_{\tau_{\beta \rho}}$ is further changed from $Q R_{\tau_{\beta \rho}}=(0.7,0.35)$ to $Q R_{\tau_{\beta \rho}}=(0.85,0.55)$, Liang et al.'s method [31] cannot deal with this estimating value and cannot derive the decision result because the adjusted loss value $Q R_{\tau_{\beta o}}=(0.85,0.55)$ is not a PFN given that $0.85^{2}+0.55^{2}>1$. Second, the basic rules of PFDTRS in Liang et al.'s method [31] are only based on the score function of PFNs, which makes Liang et al.'s method [31] unable to distinguish decision results with PFNs under some certain conditions. For instance, suppose that the expected losses for stock $z_{1}$ are derived as: $E L\left(\gamma_{\rho} \mid\left[z_{1}\right]\right)=(4 / 5,3 / 5), \quad E L\left(\gamma_{\beta} \mid\left[z_{1}\right]\right)=(3 / 5, \sqrt{2} / 5)$ and $E L\left(\gamma_{\eta} \mid\left[z_{1}\right]\right)=(7 / 10,2 / 5) . \quad$ By utilizing Liang et al.'s method [31], the score values of the expected losses for stock $z_{1}$ would be $S\left(E L\left(\beta_{\rho} \mid\left[z_{1}\right]\right)\right)=7 / 25, S\left(E L\left(\gamma_{\beta} \mid\left[z_{1}\right]\right)\right)=7 / 25, S\left(E L\left(\gamma_{\eta} \mid\left[z_{1}\right]\right)\right)=33 / 100$, which means that Liang et al.'s method [31] cannot derive the decision results.

Nevertheless, our proposed methods are able to overcome the above shortcomings. First, our proposed methods are based on $q$-ROFNs, where $q \geq 1$ and the MD, $t\left(Q R_{\tau_{*}}\right)$ and the NMD, $f\left(Q R_{\tau_{\bullet}}\right)$ of each loss value $Q R_{\tau_{\bullet}}(\bullet=\rho, \beta, \eta ; \circ=\rho, \eta)$ need to satisfy the condition that $0 \leq t\left(Q R_{\tau_{\circ}}\right)^{q}+f\left(Q R_{\tau_{\circ}}\right)^{q} \leq 1$. Thus, the adjusted loss value $Q R_{\tau_{\beta \rho}}=(0.85,0.55)$ is a $q$-ROFN, where $q$ $=3$ (or larger than 3 ) because $0.85^{3}+0.55^{3}<1$. This finding implies that our proposed methods can overcome the first drawback of Liang et al.'s method [31]. It is apparent that $q$-ROFNs have a more powerful capability to depict uncertain information of decision-making problems than IFNs and PFNs as they can express estimating loss values more completely and their application scope is wider. Second, our proposed methods are based on the score and the accuracy of $q$-ROFNs for obtaining 3 WDs, which are helpful to distinguish decision results. When $E L\left(\gamma_{\rho} \mid\left[z_{1}\right]\right)=(4 / 5,3 / 5), E L\left(\gamma_{\beta} \mid\left[z_{1}\right]\right)=(3 / 5, \sqrt{2} / 5)$ and $E L\left(\gamma_{\eta} \mid\left[z_{1}\right]\right)=(7 / 10,2 / 5)$, by utilizing our proposed method, we let $q=2$ and can obtain score values and accuracy values of the expected losses for stock $z_{1}$ as: $S\left(E L\left(\beta_{\rho} \mid\left[z_{1}\right]\right)\right)=7 / 25$, $S\left(E L\left(\gamma_{\beta} \mid\left[z_{1}\right]\right)\right)=7 / 25, \quad S\left(E L\left(\gamma_{\eta} \mid\left[z_{1}\right]\right)\right)=33 / 100 \quad, \quad H\left(E L\left(\gamma_{\rho} \mid\left[z_{1}\right]\right)\right)=1, \quad H\left(E L\left(\gamma_{\beta} \mid\left[z_{1}\right]\right)\right)=11 / 25$, $H\left(E L\left(\gamma_{\eta} \mid\left[z_{1}\right]\right)\right)=13 / 20$. Thus, $z_{1}$ is selected for investment, which implies that our proposed methods can overcome the second drawback of Liang et al.'s method [31]. Therefore, our proposed methods are more applicable to real-world decision problems.

\section{(3) Sensitivity analysis}

The main aim of sensitivity analysis is to analyse the effect of different values of parameter $q$ on the decision results of the stocks. We utilize different values of $q$ in Steps 3 and 4 of the proposed 
approach based on the first ranking technique developed in Section 5 to solve the practical Example 1.

The decision results are depicted in Figure 7. The following conclusions are drawn:

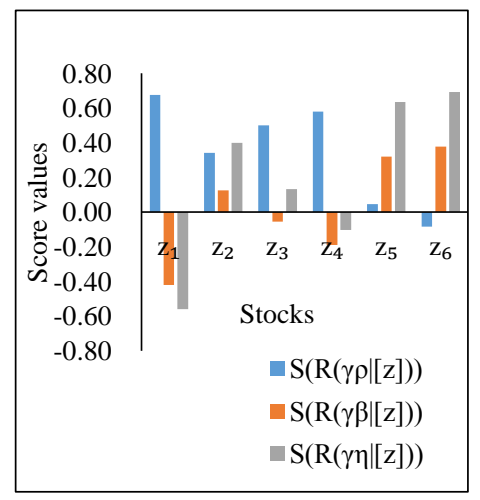

(1) $q=1$

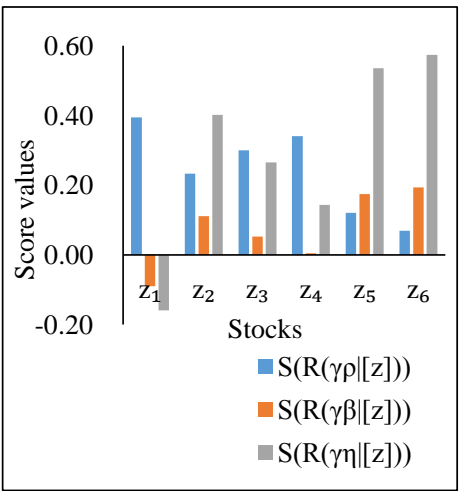

(4) $q=4$

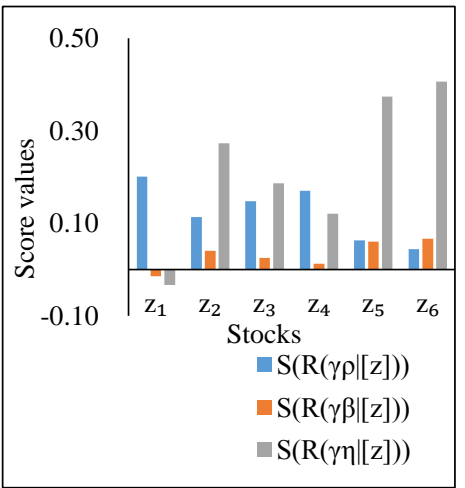

(7) $q=7$

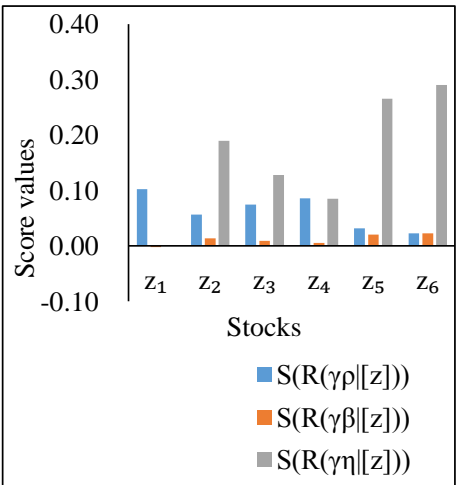

(10) $q=10$

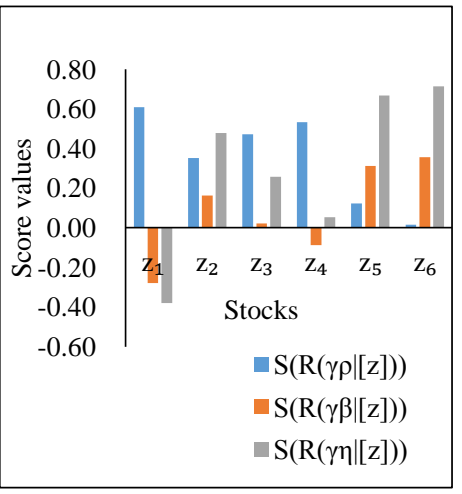

(2) $q=2$

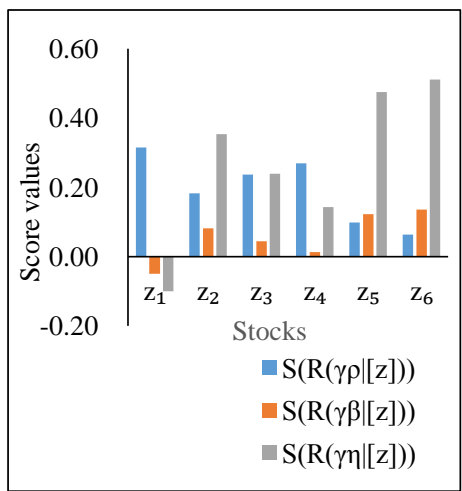

(5) $q=5$

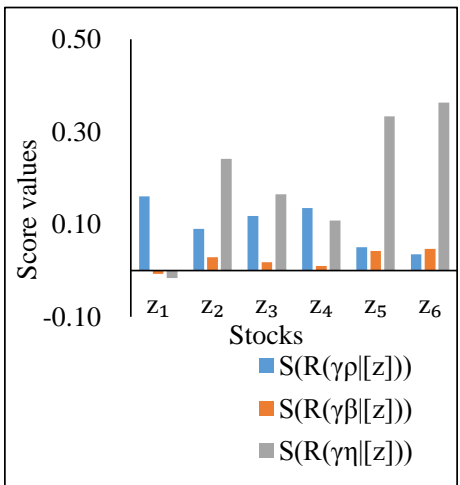

(8) $q=8$

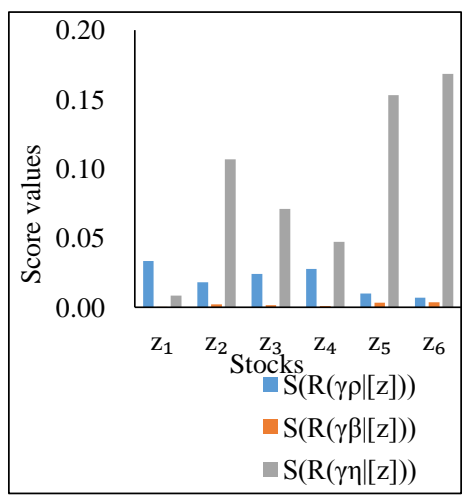

(11) $q=15$

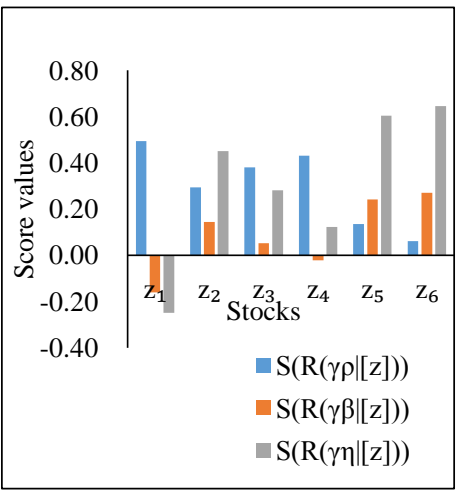

(3) $q=3$

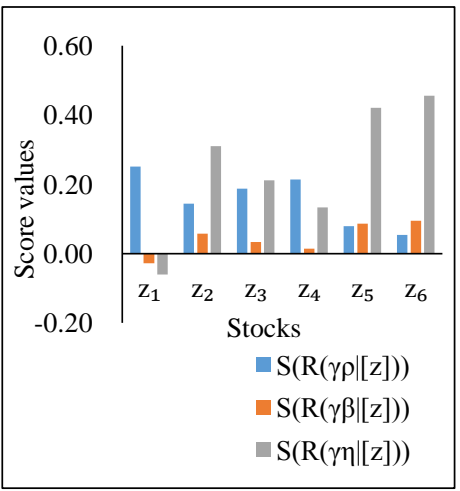

(6) $q=6$

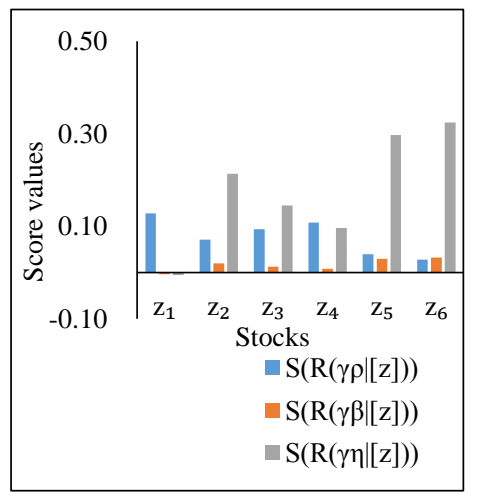

(9) $q=9$

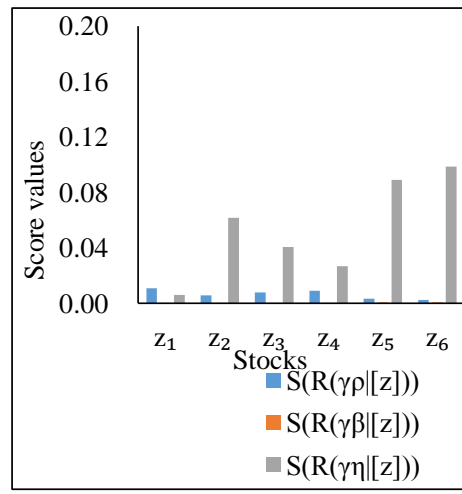

(12) $q=20$

Figure 7. Decision rules of each stock with different values of $q$. 
(1) The decision results are different with parameter $q$ increasing in the proposed method, which means the decision results are sensitive to the values of $q$. When $q=1,2, \ldots, 7$, the decision result is: $z_{1} \in \operatorname{Neg}(D), z_{2}, z_{3}, z_{4} \in \operatorname{Bou}(D)$ and $z_{5}, z_{6} \in \operatorname{Pos}(D)$; when the parameter $q=8$ or 9 , the decision result is: $z_{1} \in \operatorname{Neg}(D), z_{2}, z_{3}, z_{4}, z_{5} \in \operatorname{Bou}(D)$ and $z_{6} \in \operatorname{Pos}(D)$; when the parameter $q=10$, the decision result is: $z_{1}, z_{2}, z_{3}, z_{4}, z_{5} \in \operatorname{Bou}(D)$ and $z_{6} \in \operatorname{Pos}(D)$; and when the parameter $q=15$ or 20, the result is: $z_{1}, z_{2}, z_{3}, z_{4}, z_{5}, z_{6} \in \operatorname{Bou}(C)$. Although the decision results are different, we always obtain that $z_{2}, z_{3}$ and $z_{4}$ should be further investigated.

(2) Increasingly more stocks should be further investigated as the value $q$ increases. When $q=1$, $2, \ldots, 7, z_{2}, z_{3}, z_{4} \in \operatorname{Bou}(D) ;$ when $q=8$ or $9, z_{2}, z_{3}, z_{4}, z_{5} \in \operatorname{Bou}(D) ;$ and when $q=10$, $z_{1}, z_{2}, z_{3}, z_{4}, z_{5} \in \operatorname{Bou}(D)$; however, when the parameter $q=15$ or 20 , all the stocks need to be further investigated.

(3) All score values obtained by our proposed method approach 0 as the parameter $q$ increases.

(4) Our proposed method is more general. When $q=1, q$-ROFNs reduce to IFNs, and our proposed method based on the general ranking method becomes Liang and Liu's method [24]. Indeed, we can see that when $q=1$, the score values obtained by our proposed method (as shown in Figure 7) are the same as those of Liang and Liu's method [24] (as shown in Figure 6). When $q=2, q$-ROFNs reduce to the PFNs, and our proposed method based on the score function becomes Liang et al.'s method [31]. Thus, we can find that when $q=2$, the score values obtained by our proposed method (as shown in Figure 7) are the same as those of Liang et al.'s method [31] (as shown in Figure 6).

As shown in Figure 7, the final decision result depends on the parameter $q$. Thus, how to determine an appropriate value of the parameter $q$ is important in this problem. There are two solutions to this problem. The first way is based on experts' preferences [10]. The other one is from the viewpoint of convenience, that is, the minimum integer $q$ meeting the condition $t(Q R)^{q}+f(Q R)^{q} \leq 1$ can be taken [35]. For instance, suppose that the estimating value provided by an expert is $(0.85,0.75)$; then, we can consider the parameter $q$ attaining a value of 4 because $0.85^{3}+0.75^{3}>1$ and $0.85^{4}+0.75^{4}<1$, where the smallest integer $q$ is 4 .

\section{(4) Summary of the proposed methods}

From the above analysis, we find that the proposed methods have theoretical and managerial implications for investors. Theoretically, the methods provide a viable source to generate novel loss functions with the application of $q$-ROFSs, and subsequently provide investors with a powerful technique for dealing with the $q$-ROF decision-making problem under risks and uncertainty. In management practice, the methods are able to divide the stocks into 3WDs and inform investors regarding how to choose the action for each stock and provide the corresponding semantic explanations. In Example 1, under the minimum overall cost, 3WDs suggest that investors should invest $z_{5}$ and $z_{6} ; z_{1}$ should not be invested; and $z_{2}, z_{3}$ and $z_{4}$ should be investigated. Additionally, the proposed methods have the following advantages when compared with Liang and Liu's method [24] and Liang et al.'s 
method [31].

(1) Our proposed methods provide a wider range of fuzzy information. When $q=1, q$-ROFNs reduce to the IFNs, and our proposed method based on the first comparative method becomes Liang and Liu's method [24]. When $q=2, q$-ROFNs reduce to the PFNs, and our proposed method based on the score function becomes Liang et al.'s method [31]. Thus, our proposed methods are more general as $q$-ROFNs are a novel expansion of both IFNs and PFNs. Indeed, $q$-ROFNs incorporate the condition that the summation of the $q$ th MD and the $q$ th NMD is no more than 1 . Thus, $q$-ROFNs can not only depict the decision positions that the IFNs and PFNs can reflect but they can also depict a few other positions that the IFNs and PFNs cannot explain. Therefore, $q$-ROFNs have a more powerful capability to depict uncertain information of decision problems and thus avoid the distortion of information, which is vital to ensure the effectiveness of final decision results.

(2) Our proposed methods are more flexible. On the one hand, we propose two approaches for obtaining 3 WDs with their own features. The computational complexity of the first method is relatively inexpensive to perform, while the second method offers more information for the comparison of $q$-ROFNs via the matrix of possibility degrees. Thus, experts can flexibly select the desirable approach according to their needs and the actual decision-making problems. On the other hand, the proposed methods provide more selection choices for experts by adjusting the values of the parameter $q$, which provides another layer of flexibility.

\section{Conclusions}

In this paper, we extended the 3WDs with DTRSs to accommodate $q$-ROF settings and established a novel $q$-ROFDTRS model. Then, from different points of view, we proposed two methods to obtain 3 WDs with the novel $q$-ROFDTRS model. One is a comparative technique based on score and accuracy functions of $q$-ROFNs. The other is a comparative technique achieved by utilizing possibility degrees. At the same time, the performances of these two methods were analysed, and their corresponding features were summarized. Finally, a practical case concerning risk evaluation of a stock investment was used to analyse the practicability and viability of the new proposed methods. The outcomes relating to the proposed methods were contrasted with the results of Liang and Liu's method [24] and Liang et al.'s method [31], and the results clearly illustrate that our proposed methods generalize and handle a wider range of fuzzy information better than Liang and Liu's method [24] and Liang et al.'s method [31]. Because some parameters of our methods are given by a decision maker in advance, future research will concentrate on exploring the identification of the related parameters using machine learning techniques. Furthermore, we will concentrate on applying our research results to address some concrete practical examples in actual real-world settings, such as recommender systems [3], environmental management [50] and medical diagnosis [43].

\section{Acknowledgements}

This article was funded by the National Natural Science Foundation of China (Nos. 71771140, 71471172), 文化名家暨 “四个一批” 人才项目 (Project of cultural masters and “the four kinds of a batch" talents), and the Special Funds of Taishan Scholars Project of Shandong Province (No. 
ts201511045).

\section{References}

[1] M. Abdel-Basset, G. Manogaran, M. Mohamed, N. Chilamkurti, Three-way decisions based on neutrosophic sets and AHP-QFD framework for supplier selection problem, Future Generation Computer Systems 89 (2018) 19-30.

[2] A. Albadvi, S.K. Chaharsooghi, A. Esfahanipour, Decision making in stock trading: An application of PROMETHEE, European Journal of Operational Research 177 (2) (2007) 673-683.

[3] M. Ali, L.H. Son, N.D. Thanh, N.V. Minh, A neutrosophic recommender system for medical diagnosis based on algebraic neutrosophic measures, Applied Soft Computing 71 (2018) 1054-1071.

[4] G.R. Amin, M. Hajjami, Application of optimistic and pessimistic OWA and DEA methods in stock selection, International Journal of Intelligent Systems 31 (12) (2016) 1220-1233.

[5] K.T. Atanassov, Intuitionistic fuzzy sets, Fuzzy Sets and Systems 20 (1) (1986) 87-96.

[6] K.T. Atanassov, More on intuitionistic fuzzy sets, Fuzzy Sets and Systems 33 (1) (1989) 37-46.

[7] H. Chen, Stock selection using data envelopment analysis, Industrial Management \& Data Systems 108 (9) (2008) 1255-1268.

[8] C.C. Chen, P. Zhang, Y. Liu, J. Liu, Financial quantitative investment using convolutional neural network and deep learning technology, Neurocomputing, https://doi.org/10.1016/j.neucom.2019. 09.092.

[9] J.S. Cao, J.H. Wang, Exploration of stock index change prediction model based on the combination of principal component analysis and artificial neural network, 2019, https://doi.org /10.1007/s00500 -019-03918-3.

[10] A.P. Darko, D.C. Liang, Some $q$-rung orthopair fuzzy Hamacher aggregation operators and their application to multiple attribute group decision making with modified EDAS method, Engineering Applications of Artificial Intelligence 87 (2020) 103259, https://doi.org/10.1016/j. engappai.2019.103259.

[11] R.O. Duda, P.E. Hart, Pattern classification and Scene Analysis, Wiley, New York, 1973.

[12] M. Guo, L. Shang, Color image segmentation based on Decision-Theoretic Rough Set model and Fuzzy C-Means algorithm, IEEE International Conference on Fuzzy Systems (2014) 229-236.

[13] F. Jia, P.D. Liu, A novel three-way decision model under multiple-criteria environment, Information Sciences 471 (2019) 29-51.

[14] A. Hatami-Marbini, F. Kangi, An extension of fuzzy TOPSIS for a group decision making with an application to tehran stock exchange, Applied Soft Computing 52 (2017) 1084-1097.

[15] C.T.B Ho, K.B. Oh, Selecting Internet company stocks using a combined DEA and AHP approach, International Journal of Systems Science 41 (3) (2010) 325-336.

[16] J.H. Hu, Y. Yang, X.H. Chen, Three-way linguistic group decisions model based on cloud for medical care product investment, Journal of Intelligent \& Fuzzy Systems 33 (6) (2017) 3405-3417.

[17] W.S. Lee, G.H. Tzeng, J.L. Guan, K.T. Chien, J.M. Huang, Combined MCDM techniques for exploring stock selection based on Gordon model, Expert Systems with Applications 36 (3) (2009) 6421-6430.

[18] Y. Lee, S.H. Oh, M.W. Kim, The effect of initial weights on premature saturation in 
back-propagation learning, IJCNN-91-Seattle International Joint Conference on Neural Networks 1 (1991) 765-770

[19] H.X. Li, L.B. Zhang, X.Z. Zhou, B. Huang, Cost-sensitive sequential three-way decision modeling using a deep neural network, International Journal of Approximate Reasoning 85 (2017) $68-78$.

[20] W. Li, Y.M. Zhuang, Z.L. Ren, An extended TODIM method and its application in the stock selection under dual hesitant fuzzy linguistic information, Journal of Intelligent \& Fuzzy Systems 37 (6) (2019) 7935-7950.

[21] W.T. Li, W.H. Xu, Double-quantitative decision-theoretic rough set, Information Sciences 316 (2015) 54-67.

[22] Y. Li, C. Zhang, J. R. Swan, An information filtering model on the web and its application in JobAgent, Knowledge-Based Systems 13 (5) (2000) 285-296.

[23] D.C. Liang, D. Liu, A novel risk decision making based on decision-theoretic rough sets under hesitant fuzzy information, IEEE Transactions on Fuzzy Systems 23(2) (2015) 237-247.

[24] D.C. Liang, D. Liu, Deriving three-way decisions from intuitionistic fuzzy decision-theoretic rough sets, Information Sciences 300 (2015) 28-48.

[25] D.C. Liang, D. Liu, Systematic studies on three-way decisions with interval-valued decision-theoretic rough sets, Information Sciences 276 (2014) 186-203.

[26] D.C. Liang, D. Liu, A. Kobina, Three-way group decisions with decision-theoretic rough sets, Information Sciences 345 (2016) 46-64.

[27] D.C. Liang, D. Liu, W. Pedrycz, P. Hu, Triangular fuzzy decision-theoretic rough sets, International Journal of Approximate Reasoning 54 (2013) 1087-1106.

[28] D.C. Liang, W. Pedrycz, D. Liu, P. Hu. Three-way decisions based on decision-theoretic rough sets under linguistic assessment with the aid of group decision making, Applied Soft Computing 29 (2015) 256-269.

[29] D.C. Liang, M.W. Wang, Z.S. Xu, Heterogeneous multi-attribute nonadditivity fusion for behavioral three-way decisions in interval type-2 fuzzy environment, Information Sciences 496 (2019) 242-263.

[30] D.C. Liang, Z.S. Xu, D. Liu, Three-way decisions based on decision-theoretic rough sets with dual hesitant fuzzy information, Information Sciences 396 (2017) 127-143.

[31] D.C. Liang, Z.S. Xu, D. Liu, Y. Wu, Method for three-way decisions using ideal TOPSIS solutions at Pythagorean fuzzy information, Information Sciences 435 (2018) 282-295.

[32] S. Lim, K.W. Oh, J. Zhu, Use of DEA cross-efficiency evaluation in portfolio selection: An application to Korean stock market, European Journal of Operational Research 236 (1) (2014) 361-368.

[33] D. Liu, T.R. Li, D.C. Liang, A New Discriminant Analysis Approach under Decision-Theoretic Rough Sets, in: Proceedings of the 6th International Conference on Rough Sets and Knowledge Technology, LNAI, 6954 (2011) 476-485.

[34] D. Liu, T.R. Li, D.C. Liang, Three-way government decision analysis with decision-theoretic rough sets, International Journal of Uncertainty, Fuzziness and Knowledge-Based Systems 20 (supp01) (2012) 119-132. 
[35] P.D. Liu, S.M. Chen, P. Wang, Multiple-Attribute Group Decision-Making Based on $q$-Rung Orthopair Fuzzy Power Maclaurin Symmetric Mean Operators, IEEE Transactions on Systems, Man and Cybernetics: Systems, doi: 10.1109/TSMC.2018.2852948.

[36] P.D. Liu, P. Wang, Some $q$-Rung Orthopair Fuzzy Aggregation Operators and their Applications to Multiple-Attribute Decision Making, International Journal of Intelligent Systems 33 (2) (2018) 259-280.

[37] W. Long, Z.C. Lu, L.X. Cui, Deep learning-based feature engineering for stock price movement prediction, Knowledge-Based Systems 164 (2019) 163-173.

[38] Z. Pawlak, Rough sets, International Journal of Information and Computer Sciences 11 (1982) 341-356.

[39] M.Y. Qiu, Y. Song, F. Akagi, Application of artificial neural network for the prediction of stock market returns: The case of the Japanese stock market, Chaos, Solitons \& Fractals 85 (2016) 1-7.

[40] K.Y. Shen, M.R. Yan, G.H. Tzeng, Combining VIKOR-DANP model for glamor stock selection and stock performance improvement, Knowledge-Based Systems 58 (SI) (2014) 86-97.

[41] A. Stevanovic, B. Xue, M.J. Zhang, Feature selection based on PSO and decision-theoretic rough set model, IEEE Congress on Evolutionary Computation (2013) 2840-2847.

[42] L.V. Utkin, An imprecise extension of SVM-based machine learning models, Neurocomputing 331 (2019) 18-32.

[43] P. Wang, P.F. Zhang, Z.W. Li, A three-way decision method based on Gaussian kernel in a hybrid information system with images: An application in medical diagnosis, Applied Soft Computing 77 (2019) 734-749.

[44] C.P. Wei, X.J. Tang, A New Method for Ranking Intuitionistic Fuzzy Numbers, International Journal of Knowledge and Systems Science 2 (1) (2011) 43-49.

[45] J. Wu, F. Chiclana, A risk attitudinal ranking method for interval-valued intuitionistic fuzzy numbers based on novel score and accuracy expected functions, Applied Soft Computing 22 (2014) 272-286.

[46] J. Wu, F. Chiclana, Non-dominance and Attitudinal Prioritisation Methods for Intuitionistic and Interval-Valued Intuitionistic Fuzzy Preference Relations, Expert Systems with Applications 39 (2012) 13409-13416.

[47] J. Wu, F. Chiclana, H.C. Liao, Isomorphic multiplicative transitivity for intuitionistic and interval-valued fuzzy preference relations and its application in deriving their priority vectors, IEEE Transactions on Fuzzy Systems 26 (1) (2018) 193-202.

[48] W. Wu, W.Q. Chen, B. Liu, Prediction of ups and downs of stock market by BP neural networks, Journal of Dalian University of Technology 41 (1) (2001) 9-15.

[49] M.H. Xie, H.Y. Li, Y.J. Zhao, Blockchain financial investment based on deep learning network algorithm, Journal of Computational and Applied Mathematics 372 (2020) 112723, https://doi.org/ 10.1016/j.cam.2020.112723.

[50] P.P. Xiong, W.J. Yan, G.Z. Wang, L.L. Pe, Grey extended prediction model based on IRLS and its application on smog pollution, Applied Soft Computing 80 (2019) 797-809.

[51] R.R. Yager, Generalized orthopair fuzzy sets, IEEE Transactions on Fuzzy Systems 25 (5) (2017) 1222-1230.

[52] R.R. Yager, A.M. Abbasov, Pythagorean membership grades, complex numbers, and decision 
making, International Journal of Intelligent Systems 28 (2013) 436-452.

[53] Y.Y. Yao, An outline of a theory of three-way decisions, in Rough Sets and Current Trends in Computing, J. Yao et al., Eds. Heidelberg, Germany: Springer, 7413 (2012) 1-17.

[54] Y.Y. Yao, Granular Computing and Sequential Three-way Decisions, in: P. Lingras, et al. (Eds.), RSKT 2013, LNAI, 8171, Springer, Berlin, (2013) 16-27.

[55] Y.Y. Yao, Interval sets and three-way concept analysis in incomplete context, International Journal of Machine Learning and Cybernetics 8 (1) (2017) 3-20.

[56] Y.Y. Yao, Three-way decisions and congitive computing, Cognitive Computation 8 (4) (2016) $543-554$.

[57] Y.Y. Yao, Three-way decisions with probabilistic rough sets, Information Sciences 180 (3) (2010) 341-353.

[58] Y.Y. Yao, S.K.M. Wong, A decision theoretic framework for approximating concepts, International Journal of Man-Machine Studies 37 (6) (1992) 793-809.

[59] Y.Y. Yao, S.K.M. Wong, P. Lingras, A decision-theoretic rough set model, in: Z.W. Ras, M. Zemankova, M.L. Emrich (Eds.), Methodologies for Intelligent Systems, North-Holland, New York, 5 (1990) 17-24.

[60] H. Yu, Z.G. Liu, G.Y. Wang, An automatic method to determine the number of clusters using decision-theoretic rough set, International Journal of Approximate Reasoning 55 (1) (2014) $101-115$.

[61] J.H. Yu, B. Zhang, M.H. Chen, W.H. Xu, Double-quantitative decision-theoretic approach to multigranulation approximate space, International Journal of Approximate Reasoning 98 (2018) $236-258$.

[62] Y.H. Zhong, P.X. Zhang, Generalized Trapezoidal Decision-theoretic Rough Sets, Mathematics in Practice and Theory 45 (6) (2015) 82-88.

[63] Z.M. Zhang, A rough set approach to intuitionistic fuzzy soft set based decision making, Applied Mathematical Modelling 36 (2012) 4605-4633. 


\section{APPENDIX 1.}

Proposition 1. Suppose that $t(Q R)_{E L(\gamma,[z])}=\left(1-\left(1-t\left(Q R_{\tau_{\bullet \rho}}\right)^{q}\right)^{\operatorname{Pob}(D \mid[z])}\left(1-t\left(Q R_{\tau_{\bullet \bullet}}\right)^{q}\right)^{\operatorname{Pob}(\bar{D} \mid[z])}\right)^{1 / q}$, where $\operatorname{Pob}(D \mid[z])$ and $q$ are constant. Then, $t(Q R)_{E L\left(\gamma_{\bullet} \mid[z]\right)}$ is non-monotonic decreasing with respect to $t\left(Q R_{\tau_{\bullet}}\right)$ and $t\left(Q R_{\tau_{\bullet \eta}}\right)(\bullet=\rho, \beta, \eta)$.

Proof. Assuming that $t\left(Q R_{\tau_{\bullet} \rho}\right)$ and $t\left(Q R_{\tau_{\bullet \eta}}\right)$ are independent variables of $t(Q R)_{\left.E L\left(\gamma_{\bullet} \| z\right]\right)}$, the partial derivative of $t(Q R)_{E L\left(\gamma_{\bullet} \mid[z]\right)}$ with respect to $t\left(Q R_{\tau_{\bullet \rho}}\right)$ and $t\left(Q R_{\tau_{\bullet \eta}}\right)$, where $\operatorname{Pob}(D \mid[z]), q$ and $t\left(Q R_{\tau_{\bullet \eta}}\right)$ are constant, is as follows:

$$
\begin{aligned}
& \text { (1) } \frac{\partial t(Q R)_{E L\left(\gamma_{\bullet} \mid[z]\right)}}{\partial t\left(Q R_{\tau_{\bullet \rho}}\right)}=\left(\left(1-\left(1-t\left(Q R_{\tau_{\bullet \rho}}\right)^{q}\right)^{\operatorname{Pob}(D \mid[z])}\left(1-t\left(Q R_{\tau_{\bullet \eta}}\right)^{q}\right)^{P o b(\bar{D}[[z])}\right)^{1 / q}\right)^{\prime} \\
& =\frac{1}{q}\left(1-\left(1-t\left(Q R_{\tau_{\bullet \rho}}\right)^{q}\right)^{\operatorname{Pob}(D \mid[z])}\left(1-t\left(Q R_{\tau_{\bullet \eta}}\right)^{q}\right)^{\operatorname{Pob}(\bar{D} \mid[z])}\right)^{\frac{1-q}{q}}\left(1-\left(1-t\left(Q R_{\tau_{\bullet \rho}}\right)^{q}\right)^{\operatorname{Pob}(D \mid[z])}\left(1-t\left(Q R_{\tau_{\bullet \eta}}\right)^{q}\right)^{\operatorname{Pob}(\bar{D}[[z])}\right)^{\prime} \\
& =\frac{1}{q}\left(1-\left(1-t\left(Q R_{\tau_{\bullet \rho}}\right)^{q}\right)^{\operatorname{Pob}(D \mid[z])}\left(1-t\left(Q R_{\tau_{\bullet \eta}}\right)^{q}\right)^{\operatorname{Pob}(\bar{D} \mid[z])}\right)^{\frac{1-q}{q}}\left(1-t\left(Q R_{\tau_{\bullet}}\right)^{q}\right)^{\operatorname{Pob}(\bar{D} \mid[z])}\left(-\left(1-t\left(Q R_{\tau_{\bullet \rho}}\right)^{q}\right)^{\operatorname{Pob}(D \mid[z])}\right)^{\prime} \\
& =-\frac{\operatorname{Pob}(D \mid[z])}{q}\left(1-\left(1-t\left(Q R_{\tau_{\bullet} \rho}\right)^{q}\right)^{\operatorname{Pob}(D \mid[z])}\left(1-t\left(Q R_{\tau_{\bullet \eta}}\right)^{q}\right)^{\operatorname{Pob}(\bar{D} \mid[z])}\right)^{\frac{1-q}{q}}\left(1-t\left(Q R_{\tau_{\bullet \eta}}\right)^{q}\right)^{\operatorname{Pob}(\bar{D} \mid[z])} \\
& \times\left(1-t\left(Q R_{\tau_{\bullet} \rho}\right)^{q}\right)^{P o b(D[[z])-1}\left(1-t\left(Q R_{\tau_{\bullet} \rho}\right)^{q}\right)^{\prime} \\
& =\operatorname{Pob}(D \mid[z])\left(1-\left(1-t\left(Q R_{\tau_{\bullet \rho}}\right)^{q}\right)^{\operatorname{Pob}(D \mid[z])}\left(1-t\left(Q R_{\tau_{\bullet \eta}}\right)^{q}\right)^{\operatorname{Pob}(\bar{D} \mid[z])}\right)^{\frac{1-q}{q}}\left(1-t\left(Q R_{\tau_{\bullet \eta}}\right)^{q}\right)^{\operatorname{Pob}(\bar{D} \mid[z])} \\
& \times\left(1-t\left(Q R_{\tau_{\bullet} \rho}\right)^{q}\right)^{\operatorname{Pob}(D \mid[z])-1} t\left(Q R_{\tau_{\bullet} \rho}\right)^{q-1} \geq 0 . \\
& \text { (2) } \frac{\partial t(Q R)_{E L(\gamma, \| z])}}{\partial t\left(Q R_{\tau_{\bullet \eta}}\right)}=\left(\left(1-\left(1-t\left(Q R_{\tau_{\bullet \rho}}\right)^{q}\right)^{\operatorname{Pob}(D \|[z])}\left(1-t\left(Q R_{\tau_{\bullet \eta}}\right)^{q}\right)^{P o b(\bar{D} \mid[z])}\right)^{1 / q}\right)^{\prime} \\
& =\frac{-1}{q}\left(1-\left(1-t\left(Q R_{\tau_{\bullet} \rho}\right)^{q}\right)^{\operatorname{Pob}(D \mid[z])}\left(1-t\left(Q R_{\tau_{\bullet \eta}}\right)^{q}\right)^{\operatorname{Pob}(\bar{D}[[z])}\right)^{\frac{1-q}{q}}\left(1-t\left(Q R_{\tau_{\bullet} \rho}\right)^{q}\right)^{\operatorname{Pob}(D[[z])}\left(\left(1-t\left(Q R_{\tau_{\bullet \eta}}\right)^{q}\right)^{\operatorname{Pob}(\bar{D} \mid[z])}\right)^{\prime} \\
& =-\frac{\operatorname{Pob}(\bar{D} \mid[z])}{q}\left(1-\left(1-t\left(Q R_{\tau_{\bullet} \rho}\right)^{q}\right)^{\operatorname{Pob}(D \mid[z])}\left(1-t\left(Q R_{\tau_{\bullet \eta}}\right)^{q}\right)^{\operatorname{Pob}(\bar{D} \mid[z])}\right)^{\frac{1-q}{q}}\left(1-t\left(Q R_{\tau_{\bullet \rho}}\right)^{q}\right)^{\operatorname{Pob}(D \mid[z])} \\
& \times\left(1-t\left(Q R_{\tau_{\bullet \eta}}\right)^{q}\right)^{\operatorname{Pob}(\bar{D}[z])-1}\left(1-t\left(Q R_{\tau_{\bullet \eta}}\right)^{q}\right)^{\prime} \\
& =\operatorname{Pob}(\bar{D} \mid[z])\left(1-\left(1-t\left(Q R_{\tau_{\bullet}}\right)^{q}\right)^{\operatorname{Pob}(\bar{D} \mid[z])}\left(1-t\left(Q R_{\tau_{\bullet \rho}}\right)^{q}\right)^{\operatorname{Pob}(D \mid[z])}\right)^{\frac{1-q}{q}}\left(1-t\left(Q R_{\tau_{\bullet \rho}}\right)^{q}\right)^{\operatorname{Pob}(D \mid[z])} \\
& \times\left(1-t\left(Q R_{\tau_{\bullet \eta}}\right)^{q}\right)^{\operatorname{Pob}(\bar{D}[[z])-1} t\left(Q R_{\tau_{\bullet \eta}}\right)^{q-1} \geq 0 .
\end{aligned}
$$




\section{APPENDIX 2.}

Proposition 2. Suppose that $f(Q R)_{E L(\gamma,[z]])}=f\left(Q R_{\tau_{\bullet \rho}}\right)^{P o b(D[[z])} f\left(Q R_{\tau_{\bullet /}}\right)^{P o b(\bar{D} \mid[z])}$, where $\operatorname{Pob}(D \mid[z])$ and $q$ are constant. Then, $f(Q R)_{E L\left(\gamma_{0}[z]\right]}$ is non-monotonic decreasing with respect to $f\left(Q R_{\tau_{\bullet} \rho}\right)$ and $f\left(Q R_{\tau_{\bullet_{\eta}}}\right)(\bullet=\rho, \beta, \eta)$.

Proof. Assuming that $f\left(Q R_{\tau_{\bullet \rho}}\right)$ and $f\left(Q R_{\tau_{v_{\eta}}}\right)$ are independent variables of $f(Q R)_{E L(\gamma,[z])}$, the partial derivative of $f(Q R)_{E L(\gamma /[r])}$ with respect to $f\left(Q R_{\tau_{\sigma_{\rho}}}\right)$ and $f\left(Q R_{\tau_{\tau_{n}}}\right)$, where $\operatorname{Pob}(D \mid[z])$ and $f\left(Q R_{\tau_{\omega_{\eta}}}\right)$ are constant, is as follows:

$$
\begin{aligned}
& \text { (1) } \frac{\partial f(Q R)_{E L(\gamma,[z])}}{\partial f\left(Q R_{\tau_{\bullet_{0}}}\right)}=\left(f\left(Q R_{\tau_{\tau_{\rho}}}\right)^{P o b(D[[z])} f\left(Q R_{\tau_{\tau_{\eta}}}\right)^{P o b(\bar{D}[z]])}\right)^{\prime} \\
& =\operatorname{Pob}(D \mid[z]) f\left(Q R_{\tau_{\bullet_{\eta}}}\right)^{\operatorname{Pob}(\bar{D}[z]])} f\left(Q R_{\tau_{\bullet} \rho}\right)^{\operatorname{Pob}(D \mid[z])-1} \geq 0 .
\end{aligned}
$$

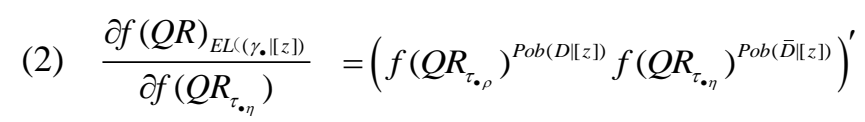

$$
\begin{aligned}
& =\operatorname{Pob}(\bar{D} \mid[z]) f\left(Q R_{\tau_{\bullet_{\rho}}}\right)^{\operatorname{Pob}(D \mid[z])} f\left(Q R_{\tau_{\bullet_{\eta}}}\right)^{\operatorname{Pob}(\bar{D}[[z])-1} \geq 0 . \\
& \text { Because } \frac{\partial f(Q R)_{E L(\gamma,[[z])}}{\partial f\left(Q R_{\tau_{\bullet \rho}}\right)} \geq 0 \text { and } \frac{\partial f(Q R)_{E L((\gamma,[[z])}}{\partial f\left(Q R_{\tau_{\bullet},}\right)} \geq 0 \text {, Proposition } 2 \text { holds. }
\end{aligned}
$$

\section{APPENDIX 3.}

Proposition 3. Suppose that $t(Q R)_{E L(\gamma,[z])}=\left(1-\left(1-t\left(Q R_{\tau_{\bullet \rho}}\right)^{q}\right)^{P o b(D[[z])}\left(1-t\left(Q R_{\tau_{\bullet}}\right)^{q}\right)^{P o b(\bar{D}[z]])}\right)^{1 / q}$, where $t\left(Q R_{\tau_{\bullet \rho}}\right), t\left(Q R_{\tau_{\bullet}}\right)(\bullet=\rho, \beta, \eta)$ and $q$ are constant. Then,

(4) If $t\left(Q R_{\tau_{\sigma_{\rho}}}\right)=1$ or $t\left(Q R_{\tau_{\sigma_{\eta}}}\right)=1, t(Q R)_{E L(\gamma /[z])}=1$.

(5) If $0 \leq t\left(Q R_{\tau_{\tau_{\eta}}}\right)<t\left(Q R_{\tau_{\omega_{\rho}}}\right)<1, t(Q R)_{E L(\gamma,[z]])}$ is monotonic increasing with respect to $P \propto[\mathbb{D}[[])$.

(6) If $0 \leq t\left(Q R_{\tau_{\bullet \rho}}\right) \leq t\left(Q R_{\tau_{\iota_{\eta}}}\right)<1, t(Q R)_{E L(\gamma,[[z])}$ is non-monotonic increasing with respect to $\operatorname{Pob}(D \mid[z])$.

Proof. If $t\left(Q R_{\tau_{\bullet \rho}}\right)=1$ or $t\left(Q R_{\tau_{\tau_{\rho}}}\right)=1$, we obtain $t(Q R)_{E L(\gamma,[z])}=1$. Suppose that $t\left(Q R_{\tau_{\sigma_{\rho}}}\right) \neq 1$ and $t\left(Q R_{\tau_{\tau_{\eta}}}\right) \neq 1$; then, the partial derivative of $t(Q R)_{E L\left(\gamma_{0}[z]\right)}$ with respect to $\operatorname{Pob}(D \mid[z])$, where $t\left(Q R_{\tau_{\omega_{\rho}}}\right), t\left(Q R_{\tau_{\tau_{\eta}}}\right)$ and $q$ are constant, is as follows:

$$
\begin{aligned}
& \frac{t(Q R)_{E L(\gamma,[z])}}{\partial \operatorname{Pob}(D \mid[z])}=\left(\left(1-\left(1-t\left(Q R_{\tau_{\sigma_{\rho}}}\right)^{q}\right)^{P o b(D \mid[z])}\left(1-t\left(Q R_{\tau_{\tau_{\eta}}}\right)^{q}\right)^{P o b(\bar{D} \mid[z])}\right)^{1 / q}\right)^{\prime} \\
& =\left(\left(1-\left(1-t\left(Q R_{\tau_{\bullet \rho}}\right)^{q}\right)^{P o b(D[z])}\left(1-t\left(Q R_{\tau_{\bullet \eta}}\right)^{q}\right)^{1-P o b(D[z])}\right)^{1 / q}\right)^{\prime}
\end{aligned}
$$




$$
\begin{aligned}
& =\frac{-1}{q}\left(1-\left(1-t\left(Q R_{\tau_{\bullet_{\rho}}}\right)^{q}\right)^{P o b(D[z])}\left(1-t\left(Q R_{\tau_{\bullet_{0}}}\right)^{q}\right)^{1-P o b(D[z])}\right)^{\frac{1-q}{q}} \\
& \times\left(\left(1-t\left(Q R_{\tau_{\bullet \rho}}\right)^{q}\right)^{P o b b(D[z]]}\left(1-t\left(Q R_{\tau_{\bullet_{0}}}\right)^{q}\right)^{1-P o b(D \mid[z])}\right)^{\prime}
\end{aligned}
$$

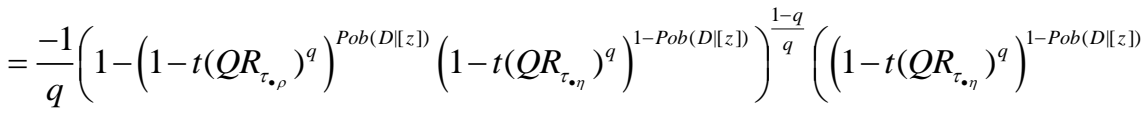

$$
\begin{aligned}
& \left.\times\left(\left(1-t\left(Q R_{\tau_{\sigma_{\rho}}}\right)^{q}\right)^{P o b(D[[z])}\right)^{\prime}+\left(1-t\left(Q R_{\tau_{\sigma_{\rho}}}\right)^{q}\right)^{P o b(D[[z])}\left(\left(1-t\left(Q R_{\tau_{\bullet_{\eta}}}\right)^{q}\right)^{1-P o b(D[[z])}\right)^{\prime}\right) \\
& =\frac{-1}{q}\left(1-\left(1-t\left(Q R_{\tau_{\bullet \rho}}\right)^{q}\right)^{P o b(D[[z])}\left(1-t\left(Q R_{\tau_{\iota_{\eta}}}\right)^{q}\right)^{1-P o b(D \mid[z])}\right)^{\frac{1-q}{q}}\left(\left(1-t\left(Q R_{\tau_{\bullet \eta}}\right)^{q}\right)^{1-P o b(D \mid[z])}\left(1-t\left(Q R_{\tau_{\bullet} \rho}\right)^{q}\right)^{P o b(D[[z])}\right. \\
& \left.\times \ln \left(1-t\left(Q R_{\tau_{\tau_{\rho}}}\right)^{q}\right)-\left(1-t\left(Q R_{\tau_{\bullet \rho}}\right)^{q}\right)^{P o b(D \mid[z])}\left(1-t\left(Q R_{\tau_{\bullet_{\eta}}}\right)^{q}\right)^{1-P o b(D \mid[z]])} \ln \left(1-t\left(Q R_{\tau_{\bullet_{\eta}}}\right)^{q}\right)\right)
\end{aligned}
$$

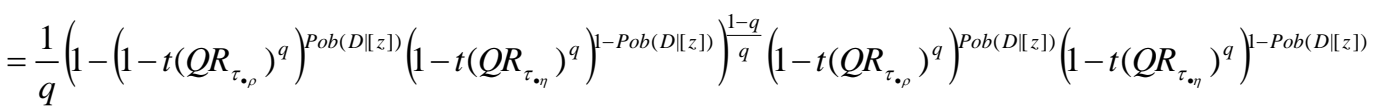

$$
\begin{aligned}
& \times \ln \frac{1-t\left(Q R_{\tau_{\bullet_{\nu}}}\right)^{q}}{1-t\left(Q R_{\tau_{\omega_{\rho}}}\right)^{q}}
\end{aligned}
$$

Because $\left(1-\left(1-t\left(Q R_{\tau_{\iota_{\rho}}}\right)^{q}\right)^{P o b(D \|[z])}\left(1-t\left(Q R_{\tau_{\bullet_{\eta}}}\right)^{q}\right)^{1-P o b(D \|[z])}\right)^{1-q} \frac{q}{q}>,\left(1-t\left(Q R_{\tau_{\iota_{\eta}}}\right)^{q}\right)^{1-P o b(D \|[z])}>0$ and $\left(1-t\left(Q R_{\tau_{\tau_{\rho}}}\right)^{q}\right)^{P o b(D[z])}>0 \quad, \quad$ when $\quad \ln \frac{1-t\left(Q R_{\tau_{\tau_{\eta}}}\right)^{q}}{1-t\left(Q R_{\tau_{\bullet \rho}}\right)^{q}}>0 \quad, \quad$ it $\quad$ is $\quad \frac{t(Q R)_{E L L(\gamma,[z])}}{\partial \operatorname{Pob}(D \mid[z])}>0 \quad$ and $t\left(Q R_{\tau_{\tau_{\eta}}}\right)<t\left(Q R_{\tau_{\tau_{\rho}}}\right)$. However, when $\quad \ln \frac{1-t\left(Q R_{\tau_{\tau_{\eta}}}\right)^{q}}{1-t\left(Q R_{\tau_{\bullet_{\rho}}}\right)^{q}} \leq 0 \quad$ it $\quad$ is $\quad \frac{t(Q R)_{E L(\gamma, \| z])}}{\partial \operatorname{Pob}(D \mid[z])} \leq 0 \quad$ and $t\left(Q R_{\tau_{\bullet \rho}}\right) \leq t\left(Q R_{\tau_{\omega_{\eta}}}\right)$. Thus, Proposition 3 holds.

\section{APPENDIX 4.}

Proposition 4. Suppose that $f(Q R)_{E L(\gamma,[z])}=f\left(Q R_{\tau_{\bullet_{\rho}}}\right)^{P o b(D \mid[z])} f\left(Q R_{\tau_{\iota_{\eta}}}\right)^{P o b(\bar{D}[z])}$, where $f\left(Q R_{\tau_{\bullet \rho}}\right)$ and $f\left(Q R_{\tau_{\bullet}}\right)(\bullet=\rho, \beta, \eta)$ are constant. Then,

(1) If $f\left(Q R_{\tau_{\bullet \rho}}\right)=0$ or $f\left(Q R_{\tau_{\tau_{\eta}}}\right)=0, f(Q R)_{\left.E L\left(\gamma_{*} \mid[z]\right]\right)}=0$.

(2) If $0<f\left(Q R_{\tau_{\nu_{\eta}}}\right)^{P o b(\bar{D} \mid[z])}<f\left(Q R_{\tau_{\bullet_{\rho}}}\right)^{P o b(D \|[z])} \leq 1, f(Q R)_{E L(\gamma,[z])}$ is monotonic increasing with respect to $\operatorname{Pob}(D \mid[z])$.

(3) If $0<f\left(Q R_{\tau_{\omega_{\rho}}}\right)^{P o b(D \mid[z])} \leq f\left(Q R_{\tau_{\omega_{\eta}}}\right)^{P o b(\bar{D} \mid[z])}<1, \quad f(Q R)_{E L(\gamma,[z])}$ is non-monotonic increasing with respect to $\operatorname{Pob}(D \mid[z])$.

Proof. If $f\left(Q R_{\tau_{\tau_{\rho}}}\right)=0$ or $f\left(Q R_{\tau_{\sigma_{\nu}}}\right)=0$, we obtain $f(Q R)_{E L(\gamma,[z]])}=0$. Suppose that $f\left(Q R_{\tau_{\sigma_{\rho}}}\right) \neq 0$ and $f\left(Q R_{\tau_{\tau_{0}}}\right) \neq 0$; the partial derivative of $f(Q R)_{E L(\gamma,[z]])}$ with respect to $\operatorname{Pob}(D \mid[z])$, where $f\left(Q R_{\tau_{\sigma_{\rho}}}\right)$ and $f\left(Q R_{\tau_{\sigma_{n}}}\right)$ are constant, is as follows: 


$$
\begin{aligned}
& \frac{\partial f(Q R)_{E L(\gamma,[z z])}}{\partial \operatorname{Pob}(D \mid[z])}=\left(f\left(Q R_{\tau_{\bullet \rho}}\right)^{P o b(D \mid[z])} f\left(Q R_{\tau_{\bullet_{\eta}}}\right)^{P o b(\bar{D} \mid[z])}\right)^{\prime} \\
& =\left(f\left(Q R_{\tau_{\bullet \rho}}\right)^{P o b(D[[z])} f\left(Q R_{\tau_{\bullet \eta}}\right)^{1-P o b(D[[z])}\right)^{\prime}
\end{aligned}
$$

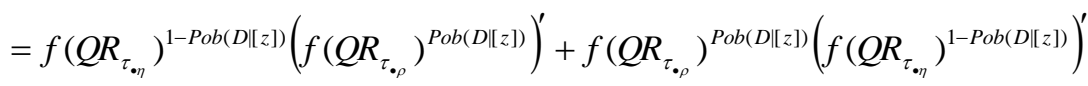

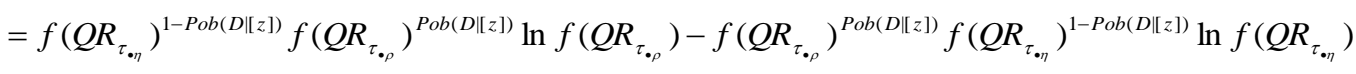

$$
\begin{aligned}
& =f\left(Q R_{\tau_{\bullet_{\eta}}}\right)^{1-P o b(D[[z])} f\left(Q R_{\tau_{\bullet \rho}}\right)^{P o b(D \|[z])} \ln \frac{f\left(Q R_{\tau_{\bullet \rho}}\right)}{f\left(Q R_{\tau_{\bullet_{\eta}}}\right)}
\end{aligned}
$$

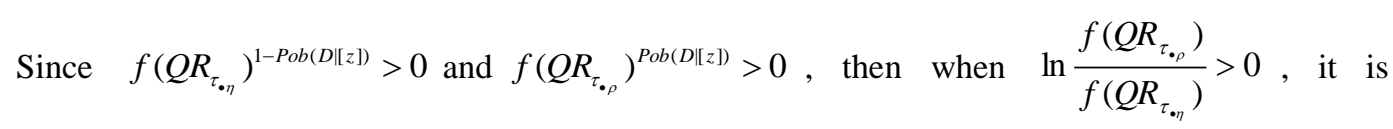
$\frac{\partial f(Q R)_{E L(\gamma, \| z])}}{\partial \operatorname{Pob}(D \mid[z])}>0 \quad$ and $\quad f\left(Q R_{\tau_{\tau_{\eta}}}\right)<f\left(Q R_{\tau_{\bullet_{\rho}}}\right)$. However, when $\ln \frac{f\left(Q R_{\tau_{\sigma_{\rho}}}\right)}{f\left(Q R_{\tau_{\iota_{\eta}}}\right)} \leq 0$, it is $\frac{\partial f(Q R)_{E L(\gamma,[z])}}{\partial \operatorname{Pob}(D \mid[z])} \leq 0$ and $f\left(Q R_{\tau_{\bullet_{\rho}}}\right) \leq f\left(Q R_{\tau_{\sigma_{\eta}}}\right)$. Thus, Proposition 4 holds. 